\title{
The evolution of the timing properties of the black-hole transient GX 339-4 during its 2002/2003 outburst ${ }^{\star}$
}

\author{
T. Belloni ${ }^{1}$, J. Homan ${ }^{1,2}$, P. Casella ${ }^{1,3,4}$, M. van der Klis ${ }^{5}$, E. Nespoli ${ }^{1}$, \\ W. H. G. Lewin' ${ }^{2}$ J. M. Miller ${ }^{6}$, and M. Méndez ${ }^{7}$ \\ 2 Center for Space Research, Massachusetts Institute of Technology, 77 Massachusetts Avenue, Cambridge, \\ 3 INAF - Osservatorio Astronomico di Roma, via di Frascati 33, 00040 Monte Porzio Catone, Italy \\ 4 Dipartimento di Fisica, Università degli Studi "Roma Tre", via della Vasca Navale 84, 00146 Roma, Italy \\ 5 Astronomical Institute "Anton Pannekoek", University of Amsterdam, and Center for High Energy Astrophysics, \\ ${ }^{6}$ Harvard-Smithsonian Center for Astrophysics, 60 Garden Street, Cambridge, MA 02138, USA \\ 7 SRON, National Institute for Space Research, Sorbonnelaan 2, 3584 CA Utrecht, The Netherlands
}

1 INAF - Osservatorio Astronomico di Brera, via E. Bianchi 46, 23807 Merate, Italy

e-mail: belloni@merate.mi .astro.it MA 02139-4307, USA Kruislaan 403, 1098 SJ, Amsterdam, The Netherlands

Received 30 November 2004 / Accepted 15 April 2005

\begin{abstract}
We present the results of the timing and color analysis of more than two hundred RXTE/PCA observations of the bright black-hole transient GX 339-4 obtained during its 2002/2003 outburst. The color-intensity evolution of the system, coupled to the properties of its fast time variability, allow the identification of four separate states. Depending on the state, strong noise is detected, together with a variety of quasi-periodic oscillations at frequencies from 0.2 to $8 \mathrm{~Hz}$. We present a characterization of the timing parameters of these states and compare them to what has been observed in other systems. These results, together with those obtained from energy spectra, point towards a common evolution of black-hole transients through their outbursts.
\end{abstract}

Key words. accretion, accretion disks - black hole physics - stars: oscillations - X-rays: binaries

\section{Introduction}

Since the early years of X-ray astronomy, black-hole candidate X-ray binaries have been known to show transitions between different spectral states. When X-ray instrumentation became sufficiently sophisticated to allow detailed timing studies on short time scales, the definitions of states were refined to include fast timing properties (see van der Klis 1995, 2005; McClintock \& Remillard 2005). The number and defining properties of these states have changed with time (see e.g. Homan et al. 2001), but it is now clear that fast timing variations are a key ingredient which needs to be considered in order to have a complete view of the states and state transitions.

Three states are relatively well identified (see McClintock \& Remillard 2005; van der Klis 2005). In the High/Soft State (HS), the energy spectrum is dominated by a soft thermal component, modeled with a disk-blackbody (Mitsuda et al. 1984 ) with a typical temperature of $\sim 1 \mathrm{keV}$. This component is attributed to an optically-thick but geometrically-thin accretion disk (Shakura \& Sunyaev 1976). Very little short-term

* Tables 1,2,4 and Appendix A are only available in electronic form at http://www. edpsciences.org variability, in the form of a power-law shaped noise component, is observed (see e.g. Belloni et al. 1999). The second is the Low/Hard State (LS): the energy spectrum is dominated by a hard component, often associated with a Comptonizing region in the accretion flow, with a high-energy cutoff energy of $\sim 100 \mathrm{keV}$. The power spectra can be decomposed in a small number of flat-top (or broad) components, approximated with Lorentzian functions, plus at times a low-frequency quasi-periodic oscillation (QPO; see Nowak 2000; Psaltis et al. 1999; Belloni et al. 2002; Pottschmidt et al. 2004). The characteristic frequencies of these components follow rather precise correlations. Low-luminosity neutron star X-ray binaries show very similar power spectra (see van der Klis 1995; Olive et al. 1998; Wijnands \& van der Klis 1999; Psaltis et al. 1999; Belloni et al. 2002; van Straaten et al. 2002, 2003), with frequencies following the same correlation, indicating a probable common origin. The third is the Quiescent state, which appears at low luminosity levels and characterizes the long periods of quiescence of transient systems. The timing properties here are not well known, given the low flux, although observations of XTE J1650-500 at a luminosity level of $\sim 10^{34} \mathrm{erg} \mathrm{s}^{-1}$ 
still suggest the presence of Low/Hard State timing properties (Tomsick et al. 2004).

In addition to these states, other behavior is observed in transient systems, often for much shorter periods. Their properties are complex and a simple classification was not possible until now. It is important to note that these additional states are not only associated to the transitions between LS and HS (see Homan et al. 2001; Belloni 2004; McClintock \& Remillard 2004; Casella et al. 2004). These states feature the largest variety of spectral and timing features. In the 1990's, a state associated to the highest luminosity observed in two systems, GS 1124-683 and GX 339-4 was called Very High State (VHS; Miyamoto et al. 1991, 1993, 1994). In this state, strong low-frequency $(1-10 \mathrm{~Hz})$ QPOs were observed together with noise variability showing rapid transitions between bandlimited and a strong power-law power-spectral shape, while the energy spectrum is composed of both a hard and a soft component, with varying relative contributions. Two different QPO behaviors were observed depending on the energy spectrum, with fast transitions between them (Takizawa et al. 1997; Miyamoto et al. 1991). Overall, two separate "flavors" of VHS were reported: a hard one, dominated by the hard component in the energy spectrum and showing band-limited noise in the power spectrum, and a soft one spectrally dominated by the soft component and with power-law noise in the power spectrum.

Later, some VHS properties were also observed at much lower luminosities and a new state was proposed, called the Intermediate State (IMS: Méndez \& van der Klis 1997; Belloni et al. 1997). Homan et al. (2001) showed that in XTE J1550564 VHS properties were found at several well-separated luminosity levels and they proposed to consider the VHS as just the highest instance of the IMS. Both HS and LS were found to occur over a wide range of luminosities, effectively removing the need to distinguish between VHS and IMS; Homan et al. (2001) proposed to consider the VHS as just the highest luminosity instance of the IMS (see also van der Klis 2005). Recently, McClintock \& Remillard (2005) proposed a new scheme for states of black-hole candidates with a somewhat different naming convention: the VHS was replaced by a more narrowly-defined Steep Power-Law state (SPL) and by several types of Intermediate States based on the parameters of the fitted spectral models. As noted by McClintock \& Remillard (2005), there is no one-to-one correspondence between these primarily X-ray spectrally defined states and the time variability states we use here.

Clearly, although there is general agreement about the LS and the HS as to their definition and their basic properties, the states that differ from those two are complex and more difficult to classify and to interpret. Notice that the LS and HS are rather stable states, which can be observed for months or years, while the remaining states are often associated to state transitions and show strong and fast variations in their properties (see e.g. Miyamoto et al. 1994; Takizawa et al. 1997; Homan et al. 2001; Nespoli et al. 2003; Casella et al. 2004). Also, the detection of high-frequency QPOs $(>20 \mathrm{~Hz})$, although few are known, seems to be limited to these states (Morgan et al. 1997; Remillard et al. 1999; Cui et al. 2000; Homan et al. 2001; Miller et al. 2001; Strohmayer 2001a,b; Homan et al. 2003a;
Klein-Wolt et al. 2004; Casella et al. 2004; Homan et al. $2005 \mathrm{~b})$. It is also in these states that narrow and often strong low-frequency $(<20 \mathrm{~Hz})$ QPOs are observed.

These QPOs have been classified into three separate classes. Wijnands et al. (1999) and Homan et al. (2001) reported on two different types of QPOs in the RXTE data of XTE J1550-564: a broad one (type A), with a quality factor $Q$ (the QPO frequency divided by the QPO full-width-halfmaximum, $F W H M$ ) of less than 3, and a narrower one (type B), with a Q larger than 6. Both QPOs were characterized by a centroid frequency of $6 \mathrm{~Hz}$ and associated with a weak red-noise component, but with different phase-lag behavior. XTE J1550 564 also showed the more common QPO-type associated with a flat-top noise component (see Cui et al. 1999 and Sobczak et al. 2000). Remillard et al. 2002 dubbed this QPO “type C": its features are a high coherence $(Q>10)$, a variable centroid frequency (in the range $0.1-10 \mathrm{~Hz}$ ) and the simultaneous presence of a strong flat-top noise component $(\sim 10-40 \% \mathrm{rms})$. The typeC QPOs are seen to follow the same global frequency correlations as those of the low/hard state (Psaltis et al. 1999; Belloni et al. 2002). While type-C QPOs are observed in many systems, the other two are less common. In addition to XTE J1550-564, type-B QPOs were also observed in GX 339-4 (Nespoli et al. 2003), GRS 1739-278 (Wijnands et al. 2001), XTE J1859+226 (Casella et al. 2004), H 1743-322 (Homan et al. 2005b) and possibly in 4U 1630-47 (Tomsick \& Kaaret 2000), while typeA QPOs were observed in GX 339-4 (Nespoli et al. 2003), XTE J1859+226 (Casella et al. 2004), H 1743-322 (Homan et al. 2003b, 2005b), and possibly in 4U 1630-47 (Tomsick \& Kaaret 2000; Dieters et al. 2000). Furthermore, in the light of the A-B-C classification, the two QPOs observed in GS 1124683 (Takizawa et al. 1997) and GX 339-4 (Miyamoto et al. 1991) can be tentatively identified with types B and C, although a detailed re-analysis of Ginga data is necessary to confirm this association. These oscillations, whose nature is still not understood, could provide a direct way to explore the accretion flow around black holes (and neutron stars). In particular, their association with specific spectral states and the phenomenology that is emerging indicate that they are a key ingredient in understanding the physical conditions that give origin to the different states.

State transitions and non LS/HS states appear to be important for the understanding of accretion onto black holes. There is evidence that the ejection of relativistic jets takes place during some state transitions (see Fender et al. 2004; Corbel et al. 2004) and it is during these intervals that structural changes take place in the accretion flow.

GX 339-4 is a transient black-hole candidate that is known to spend long periods in outburst. Historically, it was found prevalently in a hard state, although several transitions were reported (Maejima et al. 1984; Ilovaisky et al. 1986; Miyamoto et al. 1991). The system was one of the two that showed all "canonical" X-ray states of BHCs (LS, HS and VHS: see Miyamoto et al. 1991). From the launch of the Rossi X-Ray Timing Explorer (RXTE) until 1999 it remained bright, mostly in the Low/Hard state but with a transition to a softer state (see Nowak et al. 1999; Wilms et al. 1999; Belloni et al. 1999; Nowak et al. 2002; Corongiu et al. 2003). In 1999, the source 
went into quiescence, where it was detected with BeppoSAX at low flux levels (Kong et al. 2000; Corbel et al. 2003). A new outburst started in 2002 (Smith et al. 2002a,b; Nespoli et al. 2003; Belloni 2004) and ended in 2003 (Buxton \& Bailyn 2004). After roughly one year in quiescence, a new outburst started, although it has not reached the same high luminosity levels to date (Buxton et al. 2004; Smith et al. 2004; Belloni et al. 2004; Homan 2004; Kuulkers et al. 2004; Israel et al. 2004). A relativistically broadened iron emission line has been detected in the X-ray spectrum of GX 339-4, indicating the presence of a non-zero angular momentum in the black hole (Miller et al. 2004a,b).

Recently, a high mass function $\left(5.8 \pm 0.5 M_{\odot}\right)$ has been measured for the system (Hynes et al. 2003), indicating strong dynamical evidence for the black-hole nature of the compact object. The distance to GX 339-4 is not well known, with a lower limit of $\sim 6 \mathrm{kpc}$ (see Hynes et al. 2004).

GX 339-4 was the first BHC to show a radio/X-ray correlation in the LS (Hannikainen et al. 1998; Corbel et al. 2003; Markoff et al. 2003). Radio observations during the 1999 HS showed clear evidence of a strong decrease of core radio emission during this state (Fender et al. 1999b). During the 2002/2003 outburst, near the transition to the VHS (see Smith et al. 2002c), a bright radio flare was observed (Fender et al. 2002), which led to the formation of a large-scale relativistic jet (Gallo et al. 2004).

It is clear that GX 339-4 is a very important source for our understanding of the accretion and ejection properties of stellar-mass black holes. In this paper we present and discuss the results of the timing and color analysis of 205 RXTE/PCA observations of GX 339-4 during the 2002/2003 outburst. Preliminary results were presented in Belloni (2004). The results of a complete spectral analysis is presented in a forthcoming paper, while the analysis of the optical/IR/X-ray correlations is presented in Homan et al. (2005a). Spectral analysis of these data up to 2004 Jan. 29 was included in the longterm study by Zdziarski et al. (2004).

\section{Data analysis}

We analyzed a large set of 205 RXTE/PCA observations covering the 2002/2003 outburst of GX 339-4, partly from our own project and partly from data in the public archive. Notice that there are detections before MJD 52359 (see Homan et al. 2005a), but the source is too weak to detect signal in the power spectra. The observation log is shown in Table 1. The total amount of good data was $555904 \mathrm{~s}$. Since the observations correspond to a few different RXTE proposals, the observation modes differ between observations. We produced backgroundcorrected count rates for each observation in the PCA channel range 8-49 (3.8-21.2 kev), as well as a hardness ratio defined as the ratio of counts in the channel range 15-24 (6.3$10.5 \mathrm{keV})$ over those in the channel range 8-14 (3.8-6.3 keV). As the data span a long period of time, we checked the stability of the PCA gain by producing hardness and count rate from two archival observations of the Crab, from 2002 March 28 and 2003 April 23. Between these two dates, the Crab count rate was found to increase by $0.02 \%$, and its hardness to decrease by $0.2 \%$. Both values are too small to affect our results.

For timing analysis, we used custom timing-analysis software under IDL and MATLAB. For each observation we produced power spectra from stretches $128 \mathrm{~s}$ long using two separate energy bands: PCA channel band 0-35 (corresponding to $2-15.3 \mathrm{keV}$ ) for the main power spectrum, and PCA channel band $15-40(6.3-17.3 \mathrm{keV})$ in order to look for high-frequency oscillations, which are usually more prominent in this highenergy band (see Homan et al. 2002).

The power spectra in the high-energy band were also used for a consistency check on the decomposition of broad-band noise into Lorentzian components. In the following, for power spectrum we mean the low-energy power spectrum. For observations with ID 40031 the main power spectra were produced in the 8-35 $(3.8-15.3 \mathrm{keV})$ channel range for lack of data at lower energies. The chosen time resolution was $1 / 1024 \mathrm{~s}$, corresponding to a Nyquist frequency of $512 \mathrm{~Hz}$, for the lowenergy data and 1/8192 for the high-energy data. Besides examining the spectrograms, i.e. the time-frequency images, for each observation we averaged all power spectra together. The high-frequency $(>256 \mathrm{~Hz})$ part of the average power spectrum (where no signal could be seen) was fitted with a constant to estimate the level of the Poissonian noise. This average was subtracted from the power spectrum, which was then rebinned logarithmically and converted to fractional squared rms. The power spectra were then fitted with a combination of Lorentzians (see Nowak 2000; Belloni et al. 2002) using XSPEC v11.3. In the following, by flat-top Lorentzians we mean components whose centroid frequency is much lower than their width, often consistent with zero.

We also produced cross-spectra between the data in channel range $0-11(2-5 \mathrm{keV})$ and those in the channel range 12-29 $(5-12.2 \mathrm{keV})$ from stretches of 16-s length. We then calculated averaged cross-spectrum vectors for each observation, from which we derived phase-lag spectra (see Casella et al. 2004). Positive phase lags indicate that the hard curve lags the soft one. In order to extract the relevant phase-lag information for different components, we also accumulated the phase lags in specific frequency ranges: $1-64 \mathrm{~Hz}$ to characterize the full spectrum, $1-6 \mathrm{~Hz}$ for the $L_{h}$ component (see below), and for the $\mathrm{QPO}$ in a range centered on the QPO centroid frequency with a width equal to the $F W H M$ of the QPO peak itself (see Reig et al. 2000). Notice that the estimate of the QPO lags depends on the relative level of the continuum and the QPO powers in the frequency range considered. When the continuum power is significantly larger than the QPO power, the lags of the continuum may well dominate.

\section{The outburst evolution}

In this section, we describe the general evolution of the outburst, as can be followed from Figs. 1 and 2. In Fig. 1 we show the full light curve of the outburst and the corresponding evolution of the hardness. The Hardness-Intensity diagram is shown in Fig. 2. Looking at Figs. 1 and 2, we can identify four separate sections of the outburst, corresponding to the four sides of the square traced by the source in the HID. The part of the 


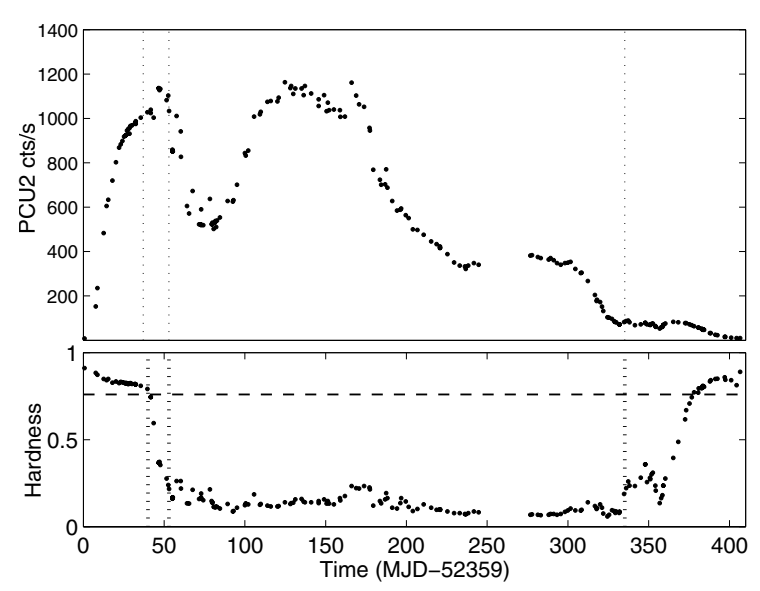

Fig. 1. Top panel: PCU2 light curve of the full outburst of GX 339-4, with one point per observation. Bottom panel: corresponding hardness ratio (see text). The dotted lines indicate major state transitions (see text). The horizontal dashed line shows the hardness level corresponding to the transition from the right branch to the left branch in the HID (see text and Fig. 2).

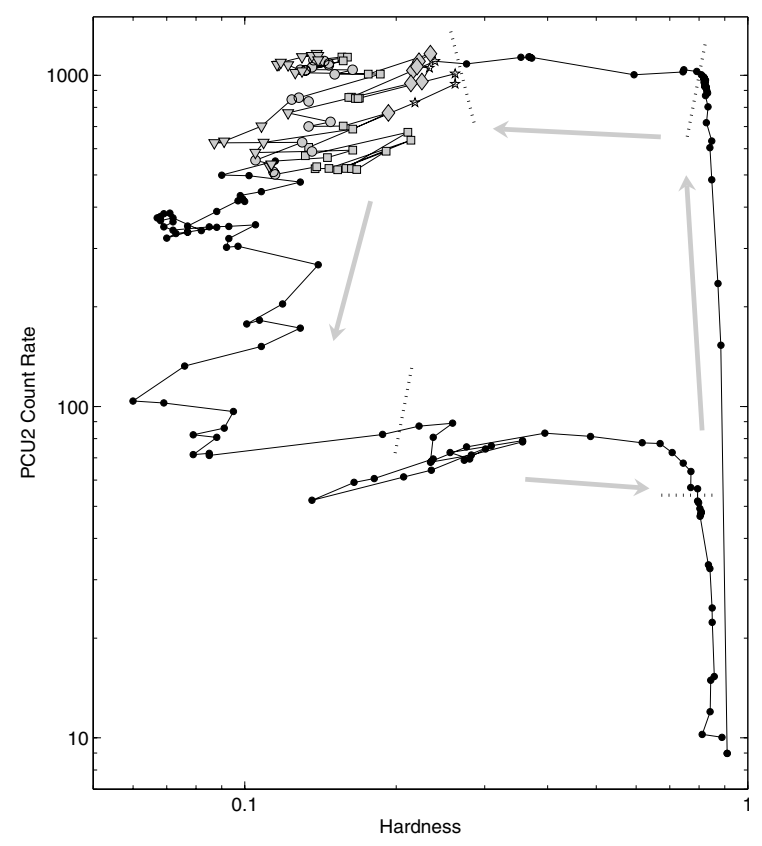

Fig. 2. Hardness-Intensity diagram corresponding to the points in Fig. 1. Each point corresponds to one observation. Dotted lines correspond to those in Fig. 1. Observation \#0 is indicated by an empty circle. The gray symbols mark the observations included in the groups B (stars), A (diamonds), U1 (squares), U2 (circles) and weak (triangles) (see Sect. 6). The gray arrows indicate the general time evolution along the outburst.

outburst covered here starts at the lower right. The source increases its flux steadily for 36 days, with only a small softening of the spectrum indicated by the slight slant of the right side of the square. Then the source on the HID starts moving rapidly to the left, with the source changing spectral shape but not its count rate. Notice that a constant count rate and a softening of the spectrum indicate that the source flux must be decreasing. The transition is indicated in both figures with a dotted line. The precise position of this line (between MJD 52394.44 and MJD 52398.66) is determined by the timing properties (see below), but also by the properties of the optical/IR/X-ray correlation, which shows a marked reversal corresponding to this date (Homan et al. 2005a). This horizontal branch lasts about 10 days, after which the left branch is reached (after MJD 52 410.53). The second transition is also shown with dotted lines: the precise positions of the transitions are determined by changes in the timing behavior (see below). There follows a long period on the left branch, which is followed with larger relative variations in hardness than on the right branch. Also, the count rate is not monotonically decreasing on this branch. After an initial motion downwards, the source moves up and reaches a count rate similar to that of the first peak, near the top of the right branch (see also Fig. 1), after which it starts decreasing again. Finally, towards the end of the outburst (after MJD 52 693.73), there is a monotonic decrease in count rate and resulting motion downwards until GX 339-4 enters the lower horizontal branch, which eventually curves and becomes once more vertical, ending its outburst on a position very close to its starting position. In order to identify these branches in terms of "canonical" BHC states, we need to investigate the timing and spectral properties. A spectral analysis will be presented in a forthcoming paper. In the following, we describe the detailed evolution of the power spectrum of GX 339-4 from a purely phenomenological point of view. The relation between our results and the source states, as determined also from spectral parameters, will be examined in the discussion.

\section{The right branch}

The first significant power detected in the power spectrum corresponds to observation \#0. Here, because of the low count rate, a fit with one Lorentzian gives a reduced $\chi^{2}$ close to unity, but the fit is clearly not good, as considerable excess power is visible at low frequencies. A fit with two Lorentzians gives as characteristic frequencies $0.021 \pm 0.7 \mathrm{~Hz}$ and $0.32 \pm 0.08 \mathrm{~Hz}$, with a total integrated fractional $\mathrm{rms}$ of $\sim 25 \%$. As these frequencies are much lower than the ones observed in subsequent observations, we do not know how to classify them and do not include them in the tables.

Observation \#1 takes place one week after Obs. \#0 and the count rate is a factor of 16 higher. The power spectrum of Obs. \#2 is shown in Fig. 3 (top spectrum). The shape of the power spectrum is very similar to that observed by RXTE in GX 339-4 during its typical Low/Hard State (see Belloni et al. 1997; Nowak et al. 1999) and also similar to that of most BHCs in that state (McClintock \& Remillard 2005). From Obs. \#1 (similar to Obs. \#2) to Obs. \#24, the power spectrum has a similar shape, with its characteristic frequencies increasing with time and therefore with count rate. All power spectra can be fitted with up to four Lorentzians: one at low frequencies $\left(L_{1}\right)$, one at intermediate frequencies $\left(L_{2}\right)$, one at high frequencies $\left(L_{3}\right)$ and a QPO peak ( $L_{Q}$, observed only in a few cases). For the first two observations, an additional Lorentzian component $L_{i}$ is needed between $L_{1}$ and $L_{2}$.

The characteristic frequencies (defined as $v_{\max }$, see Belloni et al. 2002) of these components can be seen in Table 2. Their time evolution, with the exception of the $L_{i}$ component, present 


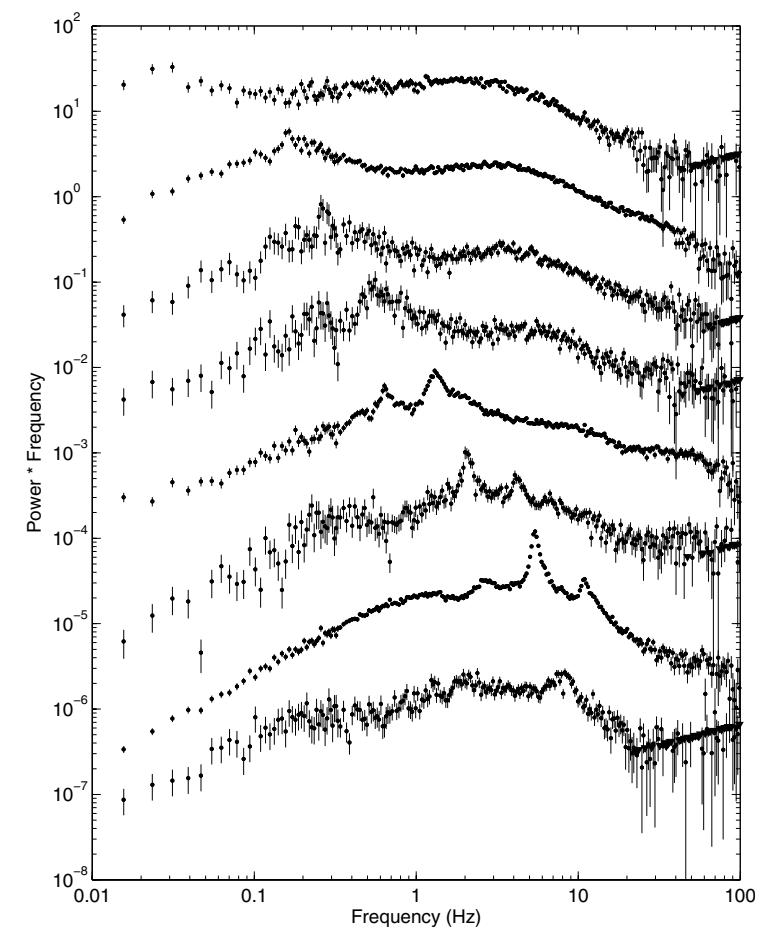

Fig. 3. Four average power spectra from the right branch and four from the top branch. From top to bottom, they correspond to observations \#2, 17, 24, 25, 27, 28, 30, 33. The power spectra are plotted in the $v P_{v}$ representation (Belloni et al. 1997) and are shifted vertically for clarity. The increasing trend at high frequencies that can be seen in some power spectra is made of $3 \sigma$ upper limits, shown as inverted triangles.

in only two spectra, can be seen in Fig. 4, together with the tight correlation between $v_{1}$ and $v_{2}$.

The integrated fractional $\mathrm{rms}$ of the $L_{1}$ component decreases smoothly from about $30 \%$ to $\sim 17 \%$, while at the same time the $L_{2}$ component goes from $28 \%$ to $14 \%$. The fractional rms of the $L_{3}$ component, when present, is always $2-3 \%$, and the few detections of $L_{Q}$ are at a level of $4-7 \%$. The quality factor $Q$ is always less than unity for $L_{1}$ and $L_{2}, \sim 1$ for $L_{3}$ and typically $\sim 8$ for $L_{Q}$.

In order to identify the broad components with those presented by Belloni et al. (2002), we need more detections of the QPO component. Therefore, this identification will be shown at the end of the next section. In any case, all the power spectra corresponding to this branch of the HID have a shape that allows us to classify these observations as belonging to the low/hard state.

The phase lags for the continuum, for the QPO, and between 1 and $6 \mathrm{~Hz}$ can be seen in Fig. 5. All lags are positive. For the QPO there are only three points, but the other two show a clear increase in the value of the hard lag from less than 0.05 radians at the start to almost 0.15 radians at the top of the right branch. A representative phase-lag spectrum (from Obs. \#8) is shown in Fig. 6. Hard lags are also typical of the low/hard state (see e.g. Pottschmidt et al. 2000).
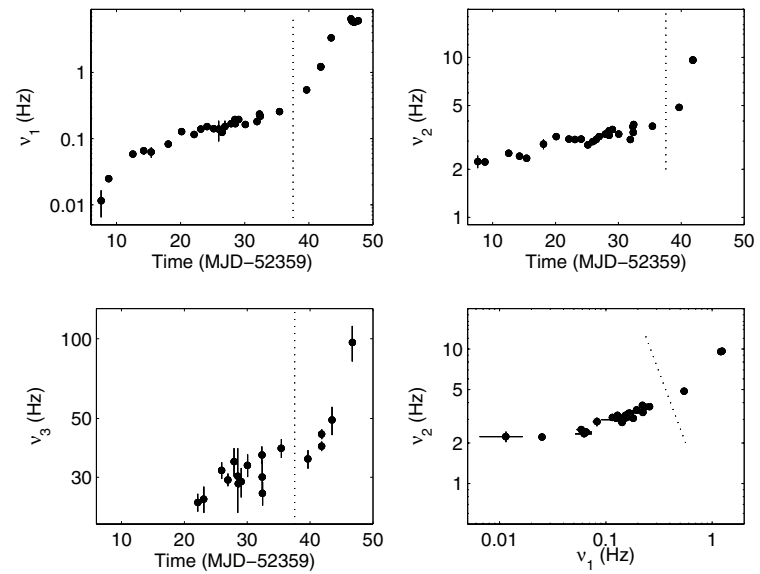

Fig. 4. First three panels: time evolution of the characteristic frequencies of the $L_{1}, L_{2}$ and $L_{3}$ components for the top/right-branch observations at the beginning of the outburst. Fourth panel: $v_{2}$ vs. $v_{1}$ correlation. In all panels, the dotted line separates the right-branch points from the top-branch points.
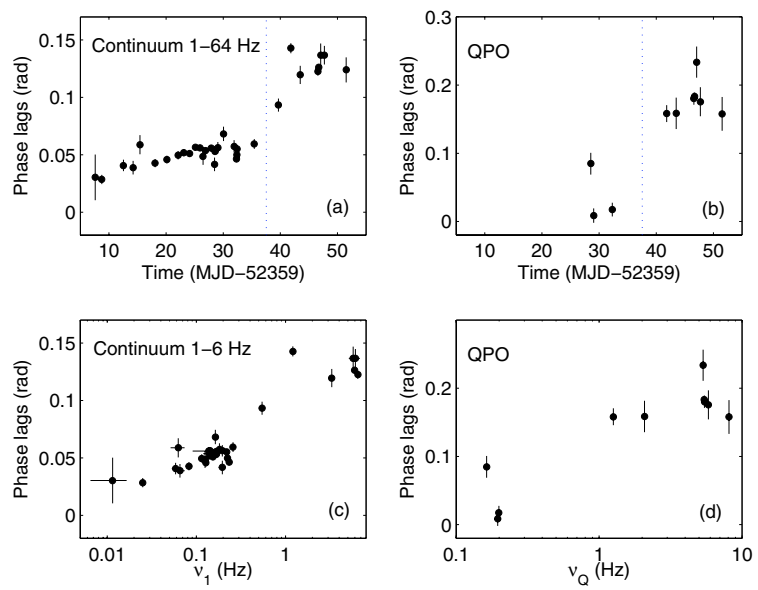

Fig. 5. Phase lags for the right and top branches. a) Time evolution of the lags of the 1-64 Hz continuum. b) Time evolution of the QPO lags. c) Correlation between the lags of the $1-6 \mathrm{~Hz}$ continuum and the characteristic frequency $v_{1}$ of the $L_{1}$ component. d) Correlation between the lags of the QPO and its characteristic frequency.

\section{The top branch}

Starting from Obs. \#25, the hardness ratio starts to decrease more rapidly (see Fig. 2) and a clear QPO with harmonic content appears in the power spectrum. This can be seen in the bottom four spectra from Fig. 3, which also shows that the characteristic frequencies continue to increase. The shape of the power spectrum is typical of the Very High State (VHS) in its hard version with band-limited noise (see Miyamoto et al. 1991, 1993, 1994; Homan \& Belloni 2005).

In order to fit these power spectra, additional components such as harmonic peaks and QPO shoulders (see e.g. Belloni et al. 1997) are needed. We decided not to include the shoulders in our fits in order to use a model consistent with that used for the previous observations. The only additional component is a broad Lorentzian $\left(L_{0}\right)$ appearing at low frequencies, while the $L_{3}$ component becomes undetectable, likely because its characteristic frequency increases and leaves the frequency 

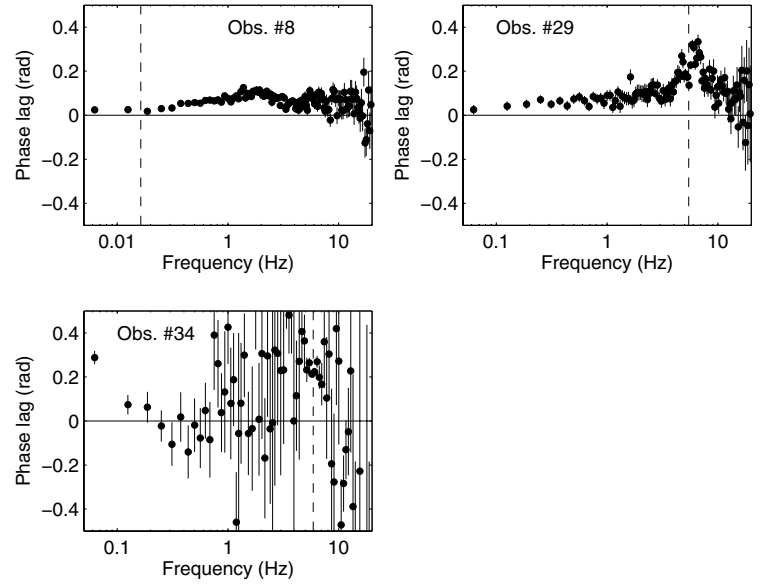

Fig. 6. Phase-lag spectra for three representative observations from the right (Obs. \#8), top (Obs. \#29) and left (Obs. \#34) branches. The vertical dashed lines mark the centroid frequency of the QPO.
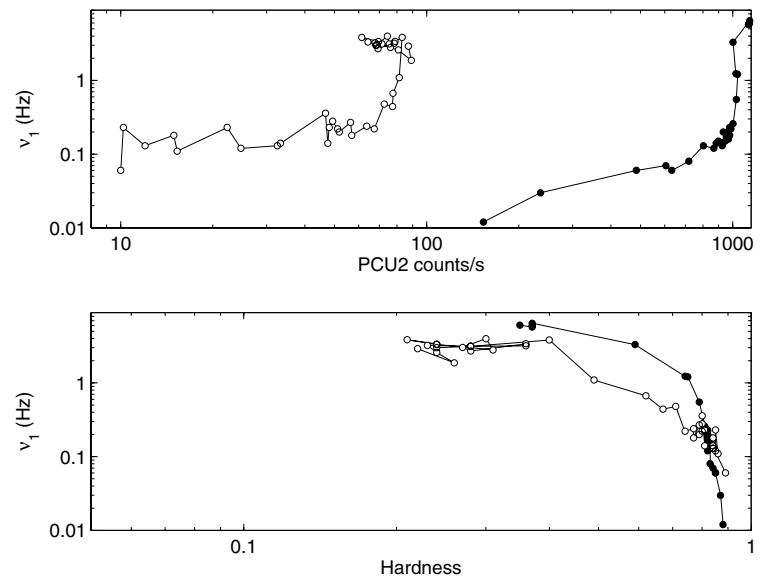

Fig. 7. Evolution of the $v_{1}$ frequency as a function of count rate (top panel) and hardness (bottom panel) for the observations of the right and top branches (filled circles) and of the bottom branch (empty circles). The $X$ axes of both panels are the same as on the corresponding axis in Fig. 1.

range where we detected significant power. The characteristic frequencies are reported in Table 2 and their evolution is shown in Fig. 4. It is evident that the components detected in the top branch are consistent with being the extension to high frequency of those seen in the right branch. However, a gradual transition can be seen in the top right panel of Fig. 4. Despite the fact that the energy spectrum changes considerably, as indicated by the hardness ratio, the timing properties do not seem to show sharp changes, with the exception of the appearance of a clear QPO of type C (see Miyamoto et al. 1994; Remillard et al. 2002; and Casella et al. 2004). The characteristic frequencies increase, and in particular $v_{1}$ and $v_{2}$ show a fast time evolution to higher values. The fourth panel in Fig. 4 shows their correlation. Figure 7 shows the evolution of $v_{1}$ for the right and top branches (filled circles) as a function of count rate and hardness. The gradual transition between the two branches is evident.

We can now attempt an identification of the components present in our power spectra and those presented in
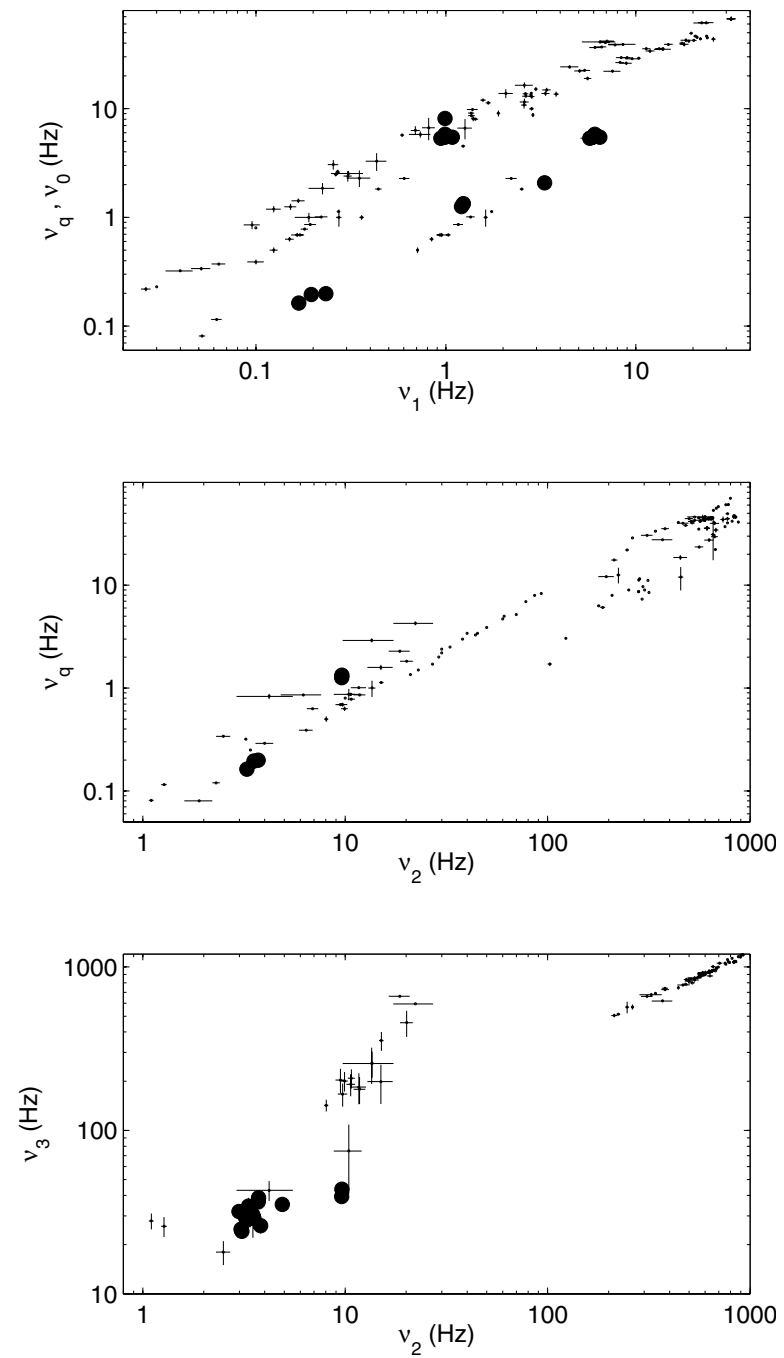

Fig. 8. Top panel: correlation between $v_{1}$ and $v_{Q}$ (large circles) and points from the WK correlation (Wijnands \& van der Klis 1999; Belloni et al. 2002). The upper points are our detections of $v_{0}$ from the top branch. Middle panel: correlation between $v_{Q}$ and $v_{2}$ (large circles) and points from the PBK correlation (Psaltis et al. 1999; Belloni et al. 2002). Bottom panel: correlation between $v_{3}$ and $v_{2}$ (large circles) and points from the PBK second correlation (Psaltis et al. 1999; Belloni et al. 2002).

Wijnands \& van der Klis (1999), Psaltis et al. (1999) and Belloni et al. (2002). It is natural to identify the $L_{Q}$ component with the low-frequency QPO in BHC. The characteristic frequency of the $L_{1}$ component is always close to $v_{Q}$ and on that basis can be identified with the $L_{h}$ component from Belloni et al. (2002). Following this scheme, $L_{0}$ corresponds to $L_{b}, L_{2}$ to $L_{\ell}$ and $L_{3}$ to $L_{u}$ (see Belloni et al. 2002, for the description of these components). In order to check whether these identifications hold, we plot the three global correlations (WK from Wijnands \& van der Klis 1999; and PBK from Psaltis et al. 1999 ) in Fig. 8, together with the corresponding points from our observations. It is worth noting that the $L_{b}\left(L_{0}\right)$ component is present here in only a few observations, while it constitutes the dominant component in other systems (see Wijnands \& van der Klis 1999). This is also seen in many power spectra of GRS 1915+105 (see e.g. Morgan et al. 1997). 
Due to this, we can put only four points on the WK relation. Also shown in Fig. 8 (top panel) are the $v_{1}-v_{Q}$ pairs, which all have $v_{1} \approx v_{Q}$. This second correlation was not present in the original Wijnands \& van der Klis (1999) work, but was presented by Belloni et al. (2002).

For comparison, from the early-outburst observations of XTE J1550-564, one can see that the $L_{b}$ and $L_{h}$ components have a rather different energy spectrum, with $L_{h}$ appearing much more prominent at higher energies (see Fig. 4 in Cui et al. 1999). However, a check from the high-energy power spectrum from our observations shows that $L_{0}$ does not appear at higher energies. Figure 8 suggests that the identification $v_{1}=v_{h}$ and $v_{2}=v_{\ell}$ is correct: in particular, the alternative interpretation $v_{1}=v_{b}$ and $v_{2}=v_{h}$ provides no match at all to the WK relation in the top panel of Fig. 8. The agreement visible in Fig. 8 shows that the identification is correct.

It is interesting to notice that the integrated fractional rms of the $L_{1}$ component continues its smooth decrease observed in the LS, reaching $\sim 8 \%$ at Obs. \#32, while the rms of $L_{2}$ drops to a few $\%$ before the component becomes undetectable. The QPO has a rather stable rms between 4-5\%, similar to that of the few detections along the right branch.

The evolution of the phase lags (see previous section) along the top branch, after the transition, can be seen in Fig. 5, while one representative phase-lag spectrum (for Obs. \#29) is shown in Fig. 6. Once again, hard lags are observed. Also the lags seem to show a smooth evolution through the state-transition. These phase lags are in marked contrast with what is usually observed in VHS observations, where the type-C QPO shows soft lags (see Wijnands et al. 1999; Reig et al. 2000; Homan et al. 2001; Remillard et al. 2002; Casella et al. 2004).

The last observation of this state, Obs. \#33, four days after Obs. \#32, shows a higher QPO frequency $(\sim 7.8 \mathrm{~Hz})$ which can be classified as type $C^{*}$ (see Casella et al. 2004). Only the $L_{0}$ component can be detected here. An additional band-limited component appears at lower frequencies, with a characteristic frequency $\sim 0.16 \mathrm{~Hz}$.

In order to compare type-C QPOs with type-B QPOs, in Fig. 9, we show the spectrogram for Obs \#30 (see Sect. 6.1). No clear time variability of the centroid frequency of the type$\mathrm{C} \mathrm{QPO}$ is seen.

\section{The left branch (top section)}

After Obs. \#33, the power spectrum changes radically and no more type-C QPOs are observed until the source leaves the left branch on the HID. As one can see from Fig. 2, after having crossed the second dotted line, GX 339-4 spends a long time to its left. During this period, the flux does not change monotonically (see Fig. 1). This complex interval continues up to observation \#109 (MJD 52 558). After this time, the PCU2 count rate remains below $500 \mathrm{cts} / \mathrm{s}$ and only a weak power-law signal is observed in the power spectrum: this bottom section of the left branch will be described in the next section. During the period before Obs. \#109, the timing behavior of GX 339-4 is complex. We summed the power spectra of consecutive observations which were close in time and had a similar position in

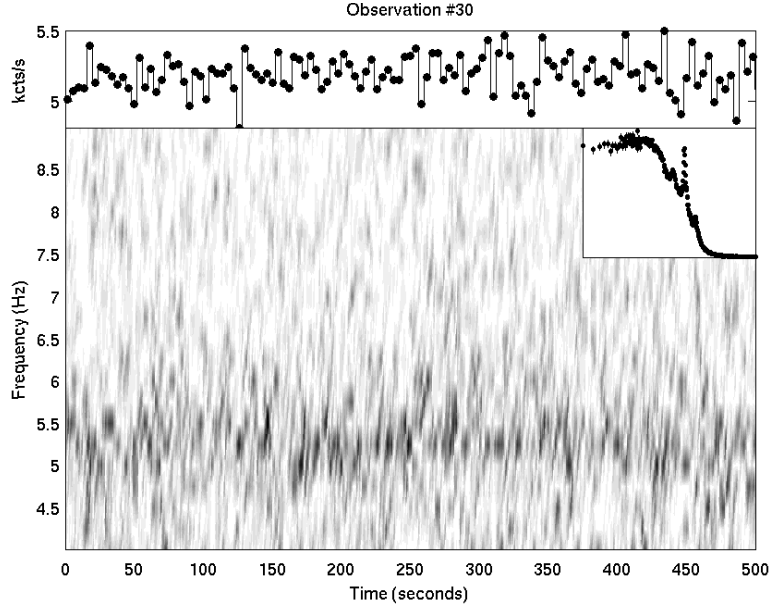

Fig. 9. Top panel: a 500-s segment of the light curve of Obs. \#30 (1 s bin size); count rate is total count rate for all PCUs. Bottom panel: corresponding spectrogram (time integration $4 \mathrm{~s}$, time step $1 \mathrm{~s}$ ), where darker points correspond to higher power. Inset: average power spectrum from the full observation.

Table 3. Log of the five types of power spectrum in the left branch. Grouped observations are indicated by "_" symbols.

\begin{tabular}{l|l}
\hline \hline Type & Observations \\
No signal & $65,68,72,73,76,77-78,83-84,89-90,100$, \\
& $105-106$ \\
Red noise & $56,57-58,60,63-64,70-71,74-75,86$ \\
Weak QPO & $44-46,66-67,69,79,82,85,88 b, 91-92,94,96$, \\
& $98-99,101-102,103 b, 107$ \\
LF noise & $35,36-40,47-50,51,52-55,59,61-62,80-81$, \\
& $87,88,93,95,103,104,108,109$ \\
type-B QPO & $34,41,42,43,97$ \\
\hline
\end{tabular}

the HID. The resulting power spectra can be summarized in a few types (see Table 3):

- no signal: no significant variability is detected in the power spectrum.

- red noise: a featureless red-noise component can be seen.

- weak QPO: typically, in the low-energy band, a broad QPO is detected at a frequency of $\sim 5 \mathrm{~Hz}$, together with a broadband noise component with a characteristic frequency of a few Hz. In the high-energy band a QPO at $\sim 10 \mathrm{~Hz}$ is also seen. All these features have an integrated fractional rms of a few $\%$.

- low-frequency noise: some low-frequency power in the form of a weak band-limited noise is observed, with no significant QPO detection either at low and high energies. Sometimes a weak QPO is also detected, making the distinction between this type and the previous one uncertain.

- type-B QPO: a narrow transient type-B QPO (see Nespoli et al. 2003) is detected during the full observation or only during a part of it. This case will be discussed in detail below.

A pattern in this complex behavior can be found by integrating the fractional $\mathrm{rms}$ in the $0.1-64 \mathrm{~Hz}$ range for all power spectra and plotting the result as a function of hardness. The result, 


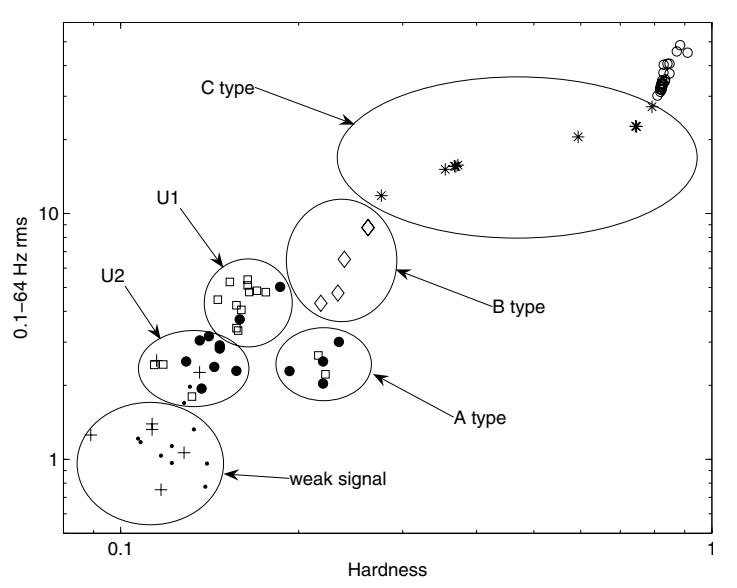

Fig. 10. Plot of integrated fractional rms versus hardness for the observations of the right, top, and upper left branch. Different symbols correspond to: right branch (open circles), top branch (asterisks), type-B QPO (diamonds), weak QPO (filled circles), LF noise (squares), red noise (plus signs) and no signal (dots). For the the definitions of the different elliptical regions, see Sect. 6.

which includes also the points from the right and top branches, can be seen in Fig. 10.

Here, right branch, top branch (type-C QPO) and type-B power spectra are clearly separated. Type-C points follow a correlation that extends all the way to the right branch points, while type-B points also follow a correlation that deviates from the type-C one, but possibly connects to it. A few observations occupy a specific region of the diagram, roughly on the extension of the type-B correlation. In analogy with what is seen in XTE J1859+226 (Casella et al. 2004) we call this region "type A" (see below). The other points are also grouped: we identify a group including almost all observations with no signal, and two additional groups that we call "U1" and "U2". These three groups correspond to different levels of integrated rms (see Fig. 10). U1 and U2 are ketp as separate sub-groups as the power spectra of most of the observations in U1 are classified as "LF noise", while those in U2 show a weak QPO. This is reflected in the average power spectra (see below). Notice that the observations corresponding to these groups are marked with different symbols in Fig. 2). In the following, we will examine the average power spectrum of these groups, and in more detail the instances of type-B QPO detections.

\subsection{Type-B QPO}

The first detection of a type-B QPO in this outburst of GX 339-4 has already been reported by Nespoli et al. (2003). The QPO in that observation (Obs \#34) is transient: during the first part of the observation only a broad bump is present in the power spectrum, while the narrow QPO appears suddenly and shows marked variations in its centroid on a characteristic time scale of $\sim 10 \mathrm{~s}$ (Nespoli et al. 2003). A similar QPO was present also in XTE J1859+226 (Casella et al. 2004). We investigated the time evolution of the QPO of the other four type-B observations by producing spectrograms with a time resolution of 4 s (see Nespoli et al. 2003) and found that indeed in all four cases the QPO is not present for the whole observation. The full

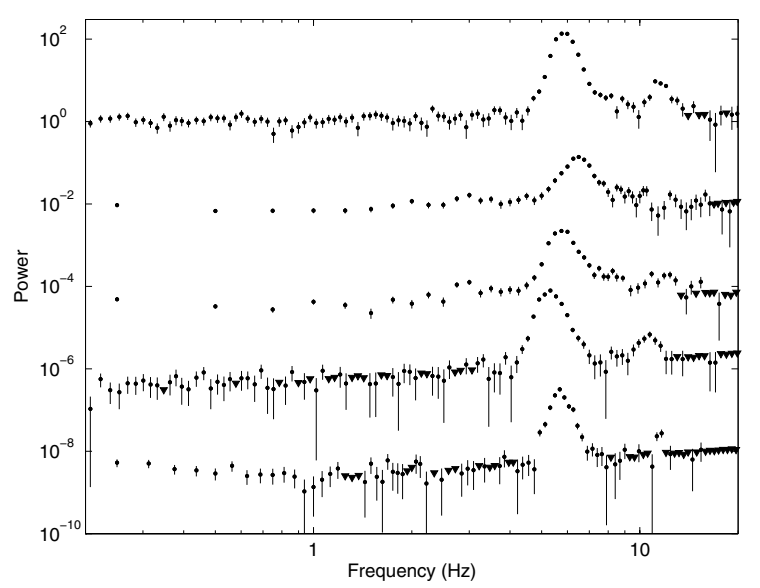

Fig. 11. The Poissonian-subtracted power spectrum of the five observations with a type-B QPO (see text), vertically shifted for clarity. From top to bottom: Obs. \#34, 41, 42, 43, 97.

description of the results for these observations can be found in Appendix A.

In summary, (transient) type-B QPOs are found in five observations (see Fig. 11): their centroid frequency ranges from 4.5 to $7.0 \mathrm{~Hz}$ and their fractional $\mathrm{rms}$ is in the range $5-8 \%$. As in the case of the type-B QPO reported by Nespoli et al. (2003), the hardness increases slightly (by $\sim 0.02$ ) when the $\mathrm{QPO}$ is present compared to the no-QPO intervals. Selecting QPO- and no-QPO intervals (see Appendix A), we can also notice that for observations \#34, 43, 97, the no-QPO $0.1-64 \mathrm{~Hz}$ $\mathrm{rms}$ is around 3\%, while when the QPO is present it is around $8-9 \%$. For the remaining two observations, \#41, 42, the noQPO and QPO fractional rms is always around 9\%, but as these are the observations where the QPO appears intermittently, our selection of the no-QPO interval was approximate and it cannot be excluded that some residual spurious power from the QPO intervals and/or from the rise and decay of the flux were included in the power spectra.

An example of the phase-lag spectrum for an observation showing a type-B QPO (Obs. \#34) can be seen in Fig. 6. The errors are large, but around the QPO frequency clear hard lags are observed, in line with what was observed in previously reported cases of type-B QPOs (see e.g. Casella et al. 2004).

\subsection{Type-A QPO}

The average power spectrum of the observations in the "type A" group (Obs. \#35, 94, 95, 96, 98-99, 103b) can be seen in Fig. 12. It can be fitted with a model consisting of two flattop Lorentzians with characteristic frequencies $0.07 \pm 0.02 \mathrm{~Hz}$ and $0.54 \pm 0.13 \mathrm{~Hz}$ respectively, plus a broad QPO with centroid $7.49 \pm 0.18 \mathrm{~Hz}$ and $F W H M 3.64 \pm 0.56 \mathrm{~Hz}$. The integrated fractional rms of the three components is $2.8 \%, 4.7 \%$ and $3.7 \%$ respectively. In the high-energy band, the power spectrum is qualitatively similar. The broad shape could be related to the averaging of different observations, but this shape is remarkably similar to that observed by Nespoli et al. (2003) in the interval before the appearance of the type-B QPO. The similarity in shape, centroid frequency and total rms make us identify 


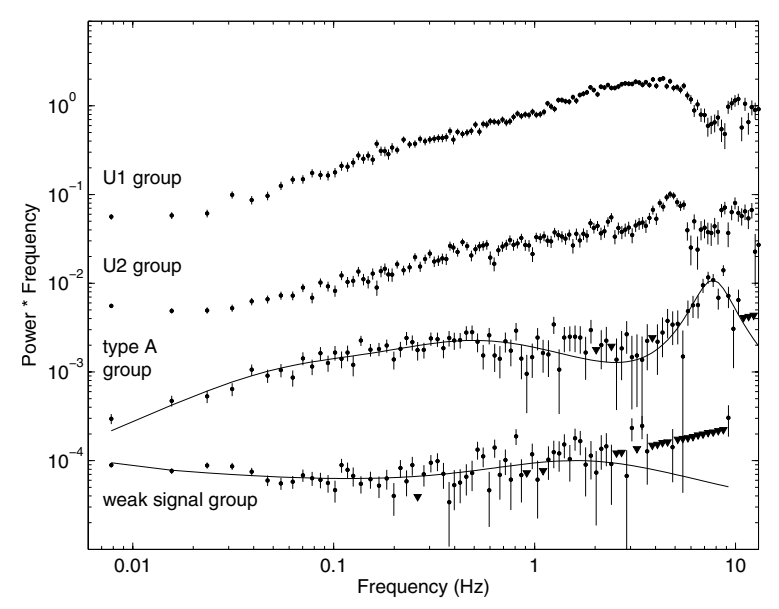

Fig. 12. Average power spectra of the observations classified as (from top to bottom) type A, weak signal, U2 and U1 (see Fig. 10). The top power spectrum is in power units, all the others are shifted downwards by a factor of 10 from each other. The lines are the best fit models described in the text.

this QPO as of type A (see Wijnands et al. 1999; Casella et al. 2004, and references therein).

\subsection{Weak signal power spectrum}

The average power spectrum of the observations in the "weak signal" group is shown in Fig. 12. Its total $0.1-64 \mathrm{~Hz}$ fractional rms is around $1.3 \%$ and can be fitted with a power law (index $1.16 \pm 0.03$ ) plus a zero-centered Lorentzian with characteristic frequency $1.75 \pm 0.33 \mathrm{~Hz}$. In the high-energy band, the power spectrum is noisy but compatible with the same shape.

\subsection{U1/U2 power spectrum}

The average power spectrum for the U1 and U2 groups appear complex (see Fig. 12). Since the exact shape could be the result of our averaging of different power spectra, we do not present detailed fits here. The U1 power spectrum shows a clear bandlimited noise component, with an excess around $10 \mathrm{~Hz}$. In the high-energy power spectrum, this excess is clearly visible as a broad QPO. The total $0.1-64 \mathrm{~Hz}$ fractional $\mathrm{rms}$ is $4.3 \%$. The $\mathrm{U} 2$ power spectrum is weaker $(2.3 \% \mathrm{rms})$ and a clear QPO is present around $5 \mathrm{~Hz}$, in addition to a band-limited noise component. A weak excess around $10 \mathrm{~Hz}$ is visible. In the high-energy band, the shape of the continuum components is similar, but the $5 \mathrm{~Hz}$ peak disappears, while the $10 \mathrm{~Hz}$ excess shows as a clear QPO peak.

\section{The left branch (bottom section)}

From observation \#110 (MJD 52560.41 ), the PCU2 count rate goes below $560 \mathrm{cts} / \mathrm{s}$ and only weak power-law noise or no noise at all is detected in the power spectrum. From Fig. 2, it is evident that all these observations are at relatively low hardness, and a much smoother path is followed mostly monotonically downwards, in the HID. We therefore accumulated one average power spectrum from these observations (\#110

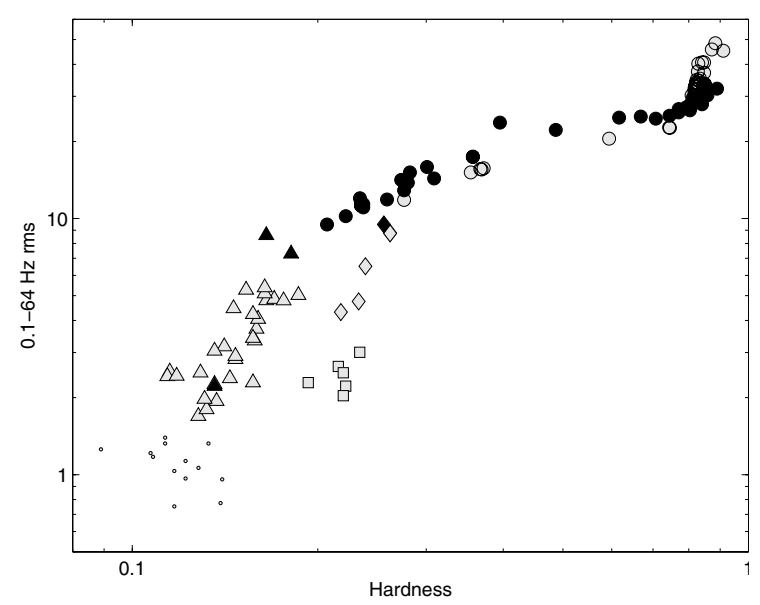

Fig. 13. Plot of integrated fractional rms versus hardness for the observations of the bottom. Open symbols represent the same points as in Fig. 10: right branch and type $\mathrm{C}$ (circles), diamonds (type B), triangles (U1/U2), squares (type a), and dots (weak signal). Filled symbols correspond to different power-spectrum shapes observed in the bottom branch (see text): flat-top noise (circles), weak noise (triangles), $1 \mathrm{~Hz}$ QPO (diamond).

to \#148f). The power spectrum is well described by a single power law with slope $0.82 \pm 0.02$. The total integrated rms between 0.1 and $64 \mathrm{~Hz}$ is only $1.6 \%$. No high-frequency cutoff is observed, with a lower limit of $25 \mathrm{~Hz}$. The average power spectrum along this branch is similar to that of the weak-signal power spectrum described above, although the power-law here is slightly flatter.

\section{The bottom branch: back to the start line}

The last observation on the left branch, with no significant signal in he power spectrum is from MJD 52693.73 (Obs. \#148f). Just before this date there is a considerable hardening (see Figs. 1 and 2). In the following observation (\#149, MJD 52 694.92), band-limited noise, often with a significant QPO, appears once again in the power spectrum. The transition is marked with a dotted line in Figs. 1 and 2 and corresponds roughly to a hardness of 0.2 .

The hardening is almost monotonic throughout the bottom branch, with the exception of a few observations that indicate a short period of softening. During three of these softer observations (Obs. \#154), the hardness went back below 0.2 and no noise components were detected in the power spectrum, indicating that the source was back in the left-branch region. For all other observations after the dotted line, noise was observed in the power spectrum. The evolution of the power spectrum can be followed once more by examining a plot of integrated fractional rms versus hardness as in Fig. 10. Figure 13 shows this diagram, where black symbols indicate observations from the bottom branch and gray symbols show the same points as in Fig. 10.

All observations indicated with circles show flat-top noise in the power spectrum, sometimes with a QPO. The three observations with hardness below 0.2 show very weak noise. One observation, located in the "type B" region from the left branch, 


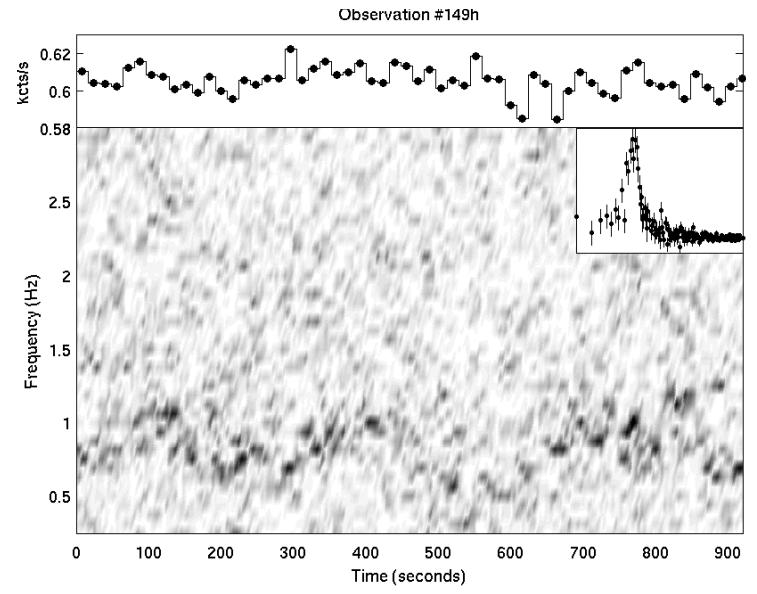

Fig. 14. Top panel: 900 s of the light curve of Obs. \#149h (16 s bin size). Bottom panel: corresponding spectrogram (time integration $16 \mathrm{~s}$, time step $4 \mathrm{~s}$ ), where darker points correspond to higher power. Inset: average power spectrum from the full observation.

shows a very peculiar power spectrum consisting essentially of a single peak at $1 \mathrm{~Hz}$. These three classes are discussed below.

\subsection{Weak noise power spectra}

These three observations with hardness below 0.2 (Obs. \#153c, $154,154 \mathrm{~b}$ ) show very little power so that it is difficult to determine the shape of the power spectrum at these low count rates. The upper limits on the rms are consistent with the value from Sect. 7.

\section{2. $1 \mathrm{~Hz} Q P O$}

Observation \#149h, on MJD 52707.91 appears completely different from the surrounding ones. The power spectrum and the corresponding spectrogram are shown in Fig. 14. The power is dominated by a single QPO peak at $0.91 \pm 0.04 \mathrm{~Hz}$, with a $F W H M$ of $0.33 \pm 0.06$. The spectrogram shows that this peak is not stable in time and shifts from 0.7 to $1.2 \mathrm{~Hz}$ over the observation. Aside from the frequency, this behavior is similar to that of the type-B QPOs described above, including the position in the color-hardness diagram. We extracted the QPO phase lag as described in the previous sections, but the results is consistent with no lags $(-0.077 \pm 0.076 \mathrm{rad})$. The fractional $\mathrm{rms}$ in the QPO peak is $\sim 7 \%$.

\subsection{Flat-top noise power spectra}

All other observations of the bottom branch, which at the end bends downwards and becomes vertical, joining the initial right branch, show rather strong noise components. Some examples are shown in Fig. 15.

We fitted the power spectra with the same model adopted for the top-branch and right-branch observations at the beginning of the outburst. Only a maximum of three components were needed (see Table 4 and Fig. 16). A narrow QPO was found to be significantly present in 10 observations. The first observations, with one exception, show only one flat-top noise

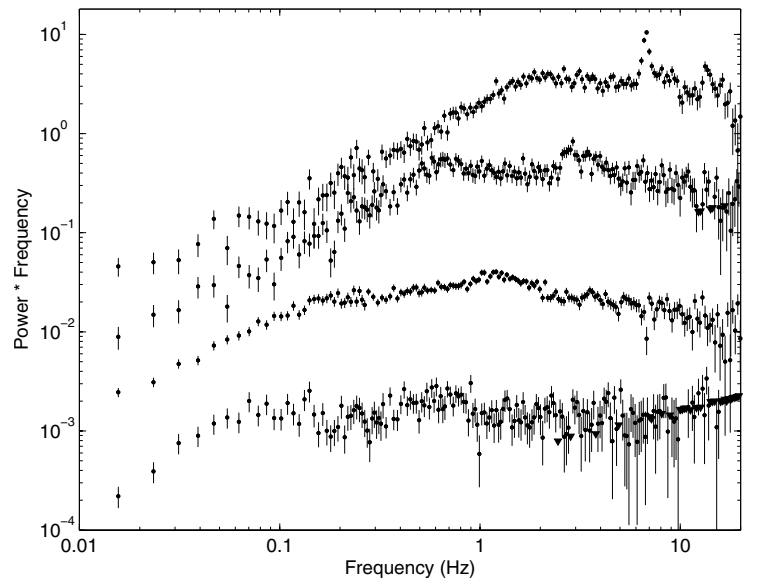

Fig. 15. Four representative power spectra from the bottom-branch observations. The time sequence here is bottom to top: observations $\# 161,158 \mathrm{~b}, 155,149 \mathrm{~g}$. The power spectra are plotted in the $v P_{v}$ representation (Belloni et al. 1997).
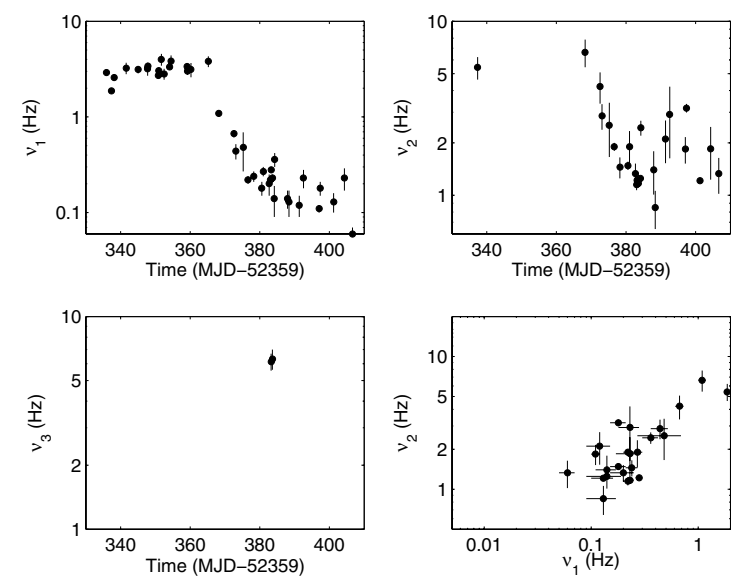

Fig. 16. Same as Fig. 4, but for the bottom-branch observations.

component. When the characteristic frequencies become low, a second noise component appears. Only in two cases it was possible to detect a third broad component. Unlike the case of the detections summarized in Table 2, the QPO frequency is substantially higher than the characteristic frequency of the first noise component, suggesting that the latter should be identified with $L_{b}$ and not with $L_{h}$ as in the previous case. This is confirmed, although with some deviations, by the plot in Fig. 17, which shows the corresponding WK correlation. The three noise components $L_{1}, L_{2}$ and $L_{3}$ in Table 4 are therefore identified as $L_{b}, L_{h}$ and $L_{\ell}$. The latter of these three identifications can only be tentative, as it is not possible to check other correlations for lack of appropriate pairs of frequencies. Comparing Figs. 4 and 16 one can see that an alternative identification could be the same as for the top branch. The evolution of $v_{1}$ as a function of count rate and hardness for the bottom branch is shown in Fig. 7 (empty circles). Clearly, it is not possible to obtain a firm identification for these components.

The integrated fractional $\mathrm{rms}$ also increases with increasing characteristic frequencies: the $L_{b}$ component goes from $8 \%$ up to $31 \%$ at the last observation, while the $L_{h}$ component goes up to $23 \%$, values similar to those at the beginning of the outburst. 


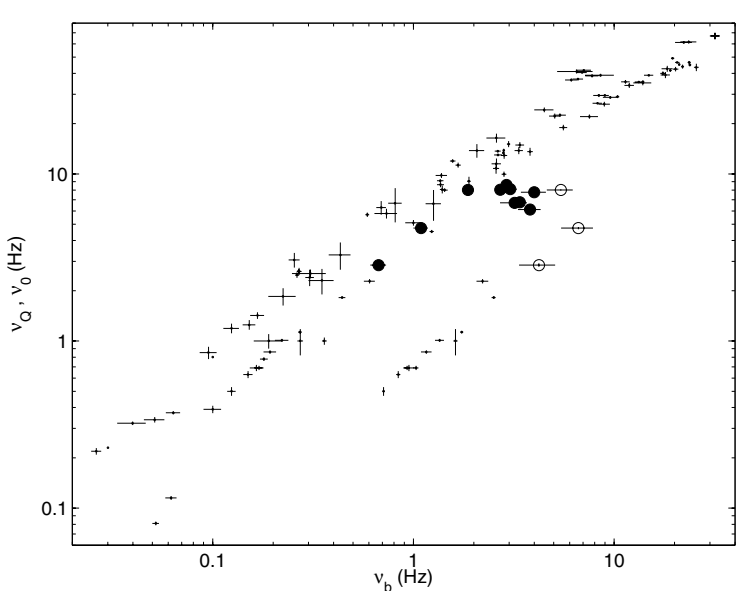

Fig. 17. WK correlation (see Wijnands \& van der Klis 1999) for the observations on the bottom branch of the HID (filled circles for $v_{b}$ and empty circles for $v_{h}$ ). The small points are from Belloni et al. (2002).

The $L_{Q}$ component has an integrated fractional rms of $4-8 \%$. For these observations at low count rate, the signal is too weak to measure phase-lags significantly different from zero.

\section{High-frequency QPOs}

No significant high-frequency peaks were seen in the single power spectra, either at low or at high energies. Since highfrequency QPOs in other systems have been detected when the source was in the IMS/VHS state, we accumulated average high-energy power spectra from all type-B and type-A observations and searched for excesses above $30 \mathrm{~Hz}$. Fits were performed to the power spectrum with a model consisting of a flat level (to account for the Poissonian component) plus a Lorentzian. The search was done fixing four values of $Q: 5$, 10,15 and 20, and with different starting centroid frequencies in order to make sure the best fit was reached. No significant peak was detected. The $3 \sigma$ one-trial upper limits for type A are $2.6 \%, 2.3 \%, 2.0 \%$ ad $1.3 \%$ for the four values of $Q$. For type B they are $2.6 \%, 2.3 \%, 2.0 \%, 2.8 \%$.

\section{Discussion}

\subsection{The outburst}

The results presented above outline a rather clear picture for the outburst of GX 339-4. From the combined timing and color properties, we can identify four main states, which have a correspondence with those determined from a detailed spectral analysis (Homan et al. in prep.) and optical/nIR data (Homan et al. 2005a). The transitions between these states correspond to precise locations in the HID and can be very fast. In the following we examine these states in terms of their timing features.

\subsubsection{The right branch: low/hard state (LS)}

The observations on the right vertical branch in Fig. 2 show hardness and timing properties typical of the "canonical" LS. The PSD is dominated by strong band-limited noise (see top four power spectra in Fig. 3). Notice the similarity between the second power spectrum from the top in Fig. 3 and that shown by Belloni et al. (1999) for the LS of GX 339-4. This noise can be decomposed into a relatively small number of Lorentzian components, which have clear counterparts in the power spectra of other systems (see Nowak 2000; Belloni et al. 2002). As usual in black-hole transients, this corresponds to the earlier part of the outburst, where the X-ray flux increases steadily (see Cui et al. 1999). Interestingly, the right branch in the HID is almost vertical, indicating that the spectral shape does not change much during the rise (see Vignarca et al. 2002; Homan et al., in prep.). In a few of the power spectra along this branch, a lowfrequency QPO is observed (see Grebenev et al. 1999; Belloni et al. 1999). All characteristic frequencies of the noise components increase as the source brightens. Hard lags increasing with time are observed along this branch, both for the continuum and the QPO (Figs. 5 and 6, see also Nowak et al. 1999).

The origin of the strong aperiodic variability observed in this state is not known. Our results confirm that in transient systems the Low/Hard state is associated to the early and late stages of the outburst, but not necessarily with low luminosities. The observed time evolution of flux and characteristic frequencies, both at the beginning and at the end of the outburst, suggests that they are correlated with mass accretion rate. The similarity in timing and color parameters indicate that the outburst ends with similar physical conditions similar to the beginning more than one year earlier.

\subsubsection{The top branch: hard intermediate state (HIMS)}

From observation \#25 (MJD 52 398), the hardness starts to decrease at a faster rate and the source enters the top branch in the HID (see Fig. 2). This horizontal path in the HID is followed considerably faster in time than the LS branch. This softening is associated with an increase of the flux from a soft thermal component, with a simultaneous steepening of the hard component (Homan et al. in prep.). As mentioned above, the transition from the right to the top branch corresponds to a marked change in the behavior of the timing parameters (see Fig. 4) and at the same time it corresponds to a clear change in the properties of the IR/optical/X-ray correlations (Homan et al. 2005a).

During this period, a clear type-C QPO appears in the power spectrum, which can be linked to that detected in some observations of the right branch. The overall noise, however, can still be fit with the same components, whose characteristic frequencies increase faster with time than on the right branch, and whose fractional $\mathrm{rms}$ is reduced (see Fig. 4). The continuum and QPO phase lags are still positive (hard lags soft) and higher than in the LS (see Figs. 5 and 6). This provides a clear link between this branch and the LS. This power-spectrum shape has been traditionally observed in the Very High State and Intermediate State of BHCs (see Belloni et al. 1997; Méndez \& van der Klis 1997), starting from the early observations of GX 339-4 and GS 1124-683 with Ginga (Miyamoto et al. 1991, 1993, 1994), when these timing properties could be associated to the presence of a relatively strong contribution from the hard component, while at softer X-ray colors, 
a different power spectrum was observed (see below). The power spectrum of the C state of GRS $1915+105$ are clearly associated to this state (see Morgan et al. 1997; Trudolyubov 2001: Reig et al. 2000). Notice that along this branch, the source does not reach the soft vertical branch yet (see Fig. 2). The end of this branch is again marked by a clear transition in the timing parameters.

We call this branch the Hard Intermediate State (see also Homan \& Belloni 2005). The fact that the timing components appear to be the same as some of those observed in the Low/Hard State suggests a similar physical origin, while the higher values for the characteristic frequencies are probably associated to a smaller value of the radius of the accretion flow associated to this time scale. This indicates that the same spectral component is responsible for this variability (see also Homan et al. in prep.).

\subsubsection{The top/left branch: soft intermediate state} (SIMS)

From Obs. \#34, as the source moves further left on the top branch, the timing properties of GX 339-4 change abruptly. On Obs. \#34, no flat-top noise component is detected, and a typeB QPO appears at $\sim 6 \mathrm{~Hz}$ (Nespoli et al. 2003). It is clear that a rather sharp transition took place here. Notice that the difference in hardness between Obs. \#33 and \#34 is small. From this observation on, GX 339-4 moves irregularly in the HID, remaining in the color range $0.05-0.25$ (see Fig. 2). No typeC QPO is detected along this branch, although we cannot at present exclude that the U1/U2 QPOs are of type C.

This branch is not as straight and monotonic as the previous ones, as the source moves up and down in flux by a large amount (see Fig. 1), and also its hardness does span a rather extended range in log space. Here, after grouping of observations together (see Sect. 6), we can identify a clear dependence of timing properties on hardness (see Fig. 10) Above $\mathrm{HR}=0.27$, the power spectra show type-C QPOs, in the HR range $0.19-0.27$ type-A and type-B QPOs, below $\mathrm{HR}=0.19$ are the U1/U2/no-signal power spectra. All these features are observed over a large range of count rates, indicating that spectral shape and not source flux is what determines the timing properties. Figure 10 indicates that the type A/B/C oscillations also correspond to different non-overlapping ranges of total $0.1-64 \mathrm{~Hz}$ rms. Notice that in the five observations where type-B QPOs are observed, the QPO is not present all the time. In the intervals without type-B QPO, in the observations where the extraction of no-QPO power spectra was not problematic, the no-QPO hardness is slightly lower and the corresponding integrated fractional $\mathrm{rms}$ drops to $\sim 3 \%$, a value that brings these observations in the type-A region of Fig. 10. The identification of these intervals with type A is strengthened by the detection of a $\sim 8 \mathrm{~Hz}$ QPO in some of those power spectra.

The characteristics of the type-B QPOs have been observed before by Ginga in the power spectrum of GS 1124-683 and GX 339-4 (see Miyamoto et al. 1991; Takizawa et al. 1997). Also fast transitions between two different types of power spectrum, corresponding to type-C and type-B QPOs, have been observed in those systems (being part of the original definition of Very Hard State), together with spectral changes. Fast transitions have been observed with RXTE in the bright transient XTE J1859+226 (Casella et al. 2004). Both type-B/type$\mathrm{A}$ and type-B/type-C transitions were observed: here a very sharp threshold in count rate was observed to correspond to the transitions. Recently, the transition between the top and the top/left branch has been identified as marking the time of the launch of relativistic outflows (see Fender et al. 2004). All these properties, together with the properties of the energy spectra of GX 339-4, lead to the conclusion that the two branches correspond to two different physical states of accretion. We call this top/left branch Soft Intermediate State (see Homan \& Belloni 2005). Notice that this state, unlike the others, is characterized by a collection of different properties in the power spectra.

Finally, softer observations along this branch are more difficult to classify. Our U1/U2/weak subdivision is the result of extensive averaging, due to the shortness of most observations.

\subsubsection{The bottom/left branch: high/soft state}

Below a PCU2 count rate of $500 \mathrm{cts} / \mathrm{s}$, corresponding to MJD $>52559$, only weak variability is observed. Looking at Fig. 2, one can see that from this time on the movement in the HID is monotonically decreasing in count rate, with a general softening. We identify this branch with the canonical High/Soft State. A long RXTE/PCA observation to GX 339-4 in this state during a previous transition showed a similar power spectrum with a slightly flatter power-law slope 0.62 (Belloni et al. 1999). Notice, however, that the observations of the upper left branch labeled "weak signal" in Fig. 10 (see Sect. 6.3) have properties that are also consistent with the High/Soft State.

\subsubsection{The bottom branch: back to the hard-intermediate and hard states}

The bottom branch corresponds to a final hardening of the spectrum. As one can see from Fig. 2, the hardening starts on the last observation of the left branch, but clear timing changes are observed only when the hardness goes above $\sim 0.2$. What we called bottom branch is clearly complex. The first part of the branch is horizontal: the source moves to the right, although it makes a significant excursion back to the region $\mathrm{HR}<0.2$. In this region, once more the power spectrum shows little noise level in the form of a power law, indicating that it was a brief interval back into the High/Soft state. In the rest of the branch however, the power spectrum shows clear flat-top band-limited noise whose characteristic frequencies follow the same correlations as those from the top and right branch. Also, Fig. 13 indicates that these power spectra show indeed the properties of the Hard Intermediate and Low/Hard States and fill the gaps in Fig. 10, suggesting a possible connection with the U1/U2 points (see Fig. 13). The major difference is that here the $L_{b}$ component clearly appears in the power spectrum, so that the source follows the main WK correlation (see Fig. 17).

Notice that the bottom branch bends smoothly and becomes vertical, ending up parallel to the initial right branch. However, 
here we do not find a sharp transition in some parameter and do not know where to put the transition to the Low/Hard state, which clearly takes place. It is interesting however to note that around MJD 52740 Obs. \#157b two things take place at the same time: the source reaches the same hardness value at which it left the LS (see Fig. 1), and the $J$-band light curve clearly shows a change (see Bailyn \& Ferrara 2004). We identify this date as the marking of the transition to the Low/Hard State (see dotted line in Fig. 2). Finally, our last point is almost exactly in the same location on the HID as our first point.

It it important to notice that, unlike what happened at high count rate in the early part of the outburst, in moving from the High/Soft state to the Hard Intermediate State, there are no observations showing clear timing features that would indicate the Soft Intermediate state, such as for instance type-A or typeB QPOs. Interestingly, there is one observation (Obs. \# 149h) which shows completely different timing properties: here the power is dominated by one single QPO peak at $\sim 1 \mathrm{~Hz}$, which shows rather large variations in time (see Fig. 14). Although no significant phase-lag estimate could be obtained, we could speculate that this is a variant of the SIMS observed at low luminosities. Clearly, more observations of this type would be needed in order to confirm this. A detailed analysis of this transition in a number of BHC is presented by Kalemci et al. (2004), who also conclude that the timing properties change more abruptly than spectral (and therefore hardness) properties. A 1-Hz QPO with similar properties was observed from XTE $\mathrm{J} 1650-500$ (Rossi et al., in preparation), and is also present in RXTE archival observations of GX 339-4.

\subsection{Source states}

In summary, the picture that emerges from the 2002/2003 outburst of GX 339-4 (see Fig. 2, Homan et al. in prep.; and Homan \& Belloni 2005) is consistent with that coming from a full spectral analysis and from multiwavelength correlations (Homan et al. 2005a). Four main regions are identified in the HID, corresponding to four separate states. These states and their transitions can be identified from the changes in the parameters of the fast time variability. The Low/Hard State (the right branch) corresponds to the first phase of the outburst and shows little spectral variations over a rather wide range of PCA count rate. Its timing properties are consistent with those usually seen in this state in GX 339-4 and in other systems.

The High/Soft State (the left branch) has a much lower hardness and small amplitude fast variability. More interesting is what happens in between these two branches, which in our data clearly corresponds to transitions between the two states mentioned above. The evolution of timing properties in the Hard Intermediate State (the top and bottom branches) can be seen as a continuation of that in the Low/Hard State and is rather similar for the high-flux and low-flux branches, corresponding to opposite transitions. A clear marker for the hardto-soft transition can be found in the timing parameters as well as in the IR/X-ray correlations, but the situation seems to be smoother for the soft-to-hard transition. Notice that this transition appears to be reversible, as for some time GX 339-4 goes back to the High/Soft state before continuing to harden to reach the Low/Hard State. During this state, type-C QPOs are observed. The phase lags of the type-C QPOs observed here are always positive, even when the centroid frequency of the QPO approaches $10 \mathrm{~Hz}$. This is in contrast to what observed in GRS 1915+105 and other transient systems (see Casella et al. 2004), indicating that the phase-lag behavior cannot be categorized in a simple way (notice that the contribution of the continuum to the phase lags can complicate its measurement, and along the top branch the $L_{h}$ component dominates it, unlike in other systems).

A fourth region (or branch) in the HID can be identified (the top/left branch), possibly present only in the highflux part of the HID. Here the timing properties are markedly different from those in the Hard Intermediate State, indicating a transition to one of the collective properties which we call Soft Intermediate State. Type-A and type-B QPOs appear and show rapid variations and transitions on short time scales. The transition to this state is very sharp and, unlike in XTE J1859+226, no more type-C QPOs are observed after it (see Casella et al. 2004) until the final part of the outburst. Interestingly, the transition into this state appears to be associated with the ejection of relativistic jets (Gallo et al. 2004; Fender et al. 2004), which again makes it an important state to study. Up to now, it has been observed in a number of systems: GX 339-4 (Miyamoto et al. 1991, 1993; Takizawa et al. 1997; Nespoli et al. 2003; this work), GS 1124-683 (Miyamoto et al. 1994), XTE J1550-564 (Homan et al. 2001; Remillard et al. 2002), XTE J1859+226 (Casella et al. 2004), H1743322 (Homan et al. 2005b), and 4U 1543-47 (Park et al. 2004), making it a rule rather than an exception. Our work shows that the behavior in this state is more complex, and additional unclassified QPOs appear (U1/U2, see above). Power spectra with similar features have been observed in other systems such as XTE J1550-564 (Remillard et al. 1999), 4U 163047 (Dieters et al. 2000; Trudolyubov et al. 2001) and possibly GRS 1915+105 (Morgan et al. 1997; Trudolyubov 2001). Clearly, these features deserve being studied in more detail with longer observations.

Interestingly, a type-B QPO has not been observed up to now in GRS 1915+105, despite the wide range of characteristics shown by this system (see Fender \& Belloni 2004, for a review). Fender et al. (2004) interpret the X-ray/radio correlations of GRS 1915+105 and other transients in the framework of the same model; however, the time scales involved in GRS $1915+105$ are much shorter and it is possible that the source moves too fast through its HID to show the timing features of this state. A full comparative analysis of a number of transient systems will be presented in a forthcoming paper.

The state paradigm presented here is not the same as that outlined in McClintock \& Remillard (2005). Although the Low/Hard and High/Soft state can obviously be identified with the Hard and Thermal-Dominant states, our two additional states are not the same as the Steep-Power-Law and Intermediate-State of McClintock \& Remillard (2005). A precise comparison is not possible, since their classification is based on model-dependent parameters coming from spectral fits, but the variety of power spectra shown to be associated 
to the SPL in McClintock \& Remillard (2005), which includes both type-A/B and type-C QPOs, excludes a one-to-one relation to ours.

\subsection{Quasi-periodic oscillations}

With these results, the $\mathrm{A} / \mathrm{B} / \mathrm{C}$ classification of low-frequency QPOs in black-hole candidates is strengthened. There are still undecided cases such as our U1/U2 power density spectra, but they all correspond to low signal-to-noise signals that might turn out to belong to one of the three types once observed with higher statistics.

Type-C QPOs are the most common and are observed in all systems that show LS and HIMS (see McClintock \& Remillard 2005; van der Klis 2005). Their properties are rather well defined. They are clearly associated to the hard component in the energy spectrum (see e.g. Casella et al. 2004) and appear during states when the hard component shows a high-energy cutoff and is possibly associated to an outflow (see Fender et al. 2004). Their frequency is strongly correlated with other observed properties such as the source flux and X-ray color. They are associated to the QPOs observed sporadically in the LS, and a similar association can be made between the broad-band noise properties of HIMS and LS. There is no consensus as to the physical origin of type-C QPOs, but it is clear that these oscillations are particularly important for our understanding of the accretion flow, as they relate to a spectral component that could be associated to an outflow from the system (see Kanbach et al. 2001; Rodriguez et al. 2004).

QPOs of types A and B are detected over a much smaller range of frequencies (see also Casella et al. 2004) and, while having a similarly hard energy spectrum, are most likely associated to a different hard component, for which no highenergy cutoff is observed (see Zdziarski et al. 2001). Rodriguez et al. (2004), using simultaneous RXTE/Integral observation, showed that for GRS 1915+105, the energy dependence of the type-C QPO indicates the presence of a high-energy cutoff, while the energy spectrum is composed of two separate components. These type-A/B oscillations appear in soft intervals of the HID, where no compact jet emission is observed in the radio (Fender et al. 2004). The fast transitions observed between these two types of QPO and in particular transitions involving also the type-C QPO (Casella et al. 2004) could be indicative of short transitions to the HIMS. These mini-transitions could be responsible for small jet ejections similar to those observed on short (hour) time scales in GRS 1915+105 (Fender \& Belloni 2004; Fender et al. 2004). These transitions are indeed related to variations in hardness (GX 339-4) or intensity (XTE J1859+226). Their limited range of frequencies, especially of the type-B QPOs is particularly interesting. Since a transient oscillation at a similar frequency has been observed in the neutron star system 4U 1820-30 (Belloni et al. 2004), if this association is correct the frequency of type-B QPOs should have a weak dependence on the mass of the compact object. Notice that the $1 \mathrm{~Hz}$ QPO detected near the end of the outburst appears at a hardness value of 0.26 , comparable to that at which the $6 \mathrm{~Hz}$ type-B QPOs are seen. Since the properties of this $1 \mathrm{~Hz}$ oscillation (besides its centroid frequency) are compatible with those of type-B QPOs, it is possible that the small range of the frequency of these QPOs is due to their appearing only at relatively bright phases of the outburst. In other words, there is also a count rate dependency of the frequency of type-B QPOs, but most of these features appear at a high count rate. In our data, there is some evidence of a count rate dependence of the $6 \mathrm{~Hz}$ QPO, as it is in the case of XTE J1859+226 (Casella et al. 2004). At any rate, the fast transitions between QPO types observed here (and in other systems) indicate that state transitions take place on very short time scales and are clearly traced by the timing properties. The three types of QPO described here $(\mathrm{A} / \mathrm{B} / \mathrm{C})$ are rather different and are possibly tracers of separate spectral components.

\section{Summary and conclusions}

We analyzed a large set of RXTE/PCA observations of GX 339-4 during its complex 2002/2003 outburst. From the timing and color results, we identify four main states of the source, with a distinctive pattern of evolution of the outburst. The timing characteristics during the outburst are complex, but show simultaneous spectral transitions that allow a clear categorization. These states (in time sequence) are:

- Low/Hard state: this state is associated with relatively low values of the accretion rate, i.e. lower than in the other bright states, although it is clearly not limited to low flux intervals. The energy spectrum is hard and the fast time variability is dominated by a strong ( $30 \%$ fractional $\mathrm{rms})$ band-limited noise. Sometimes, low frequency QPOs are observed. The characteristic frequencies detected in the power spectra follow broad-range correlations (see Belloni et al. 2002).

- Hard Intermediate state: in this state, the energy spectrum is softer than in the LS. The power spectra feature bandlimited noise with characteristic frequency higher than the LS and a rather strong $1-8 \mathrm{~Hz}$ type-C QPO. The frequencies of the main components detected in the power spectra extend the broad correlations mentioned for the LS. However, a sharp transition from the LS can be identified by looking at the IR/X-ray correlation (Homan et al. 2005a).

- Soft Intermediate state: here the energy spectrum is systematically softer than the HIMS. No strong band-limited noise is observed, but transient type-A and type-B QPOs appear, the frequency of which spans only a limited range. The transition from the HIMS is very sharp and marked by the transition from type-C to type-A/B QPOs in the power spectra, despite the relatively small changes in hardness. This state is characterized by a variety of timing properties, of which type-A/B QPOs are only a subset.

- High/Soft state: the energy spectrum is very soft. Only weak power-law noise is observed in the power spectrum. The onset of this state is identified with the systematic nondetection of features in the power spectrum.

After the HS, a transition back to the HIMS is observed, marked by the sudden appearance of band-limited noise with 


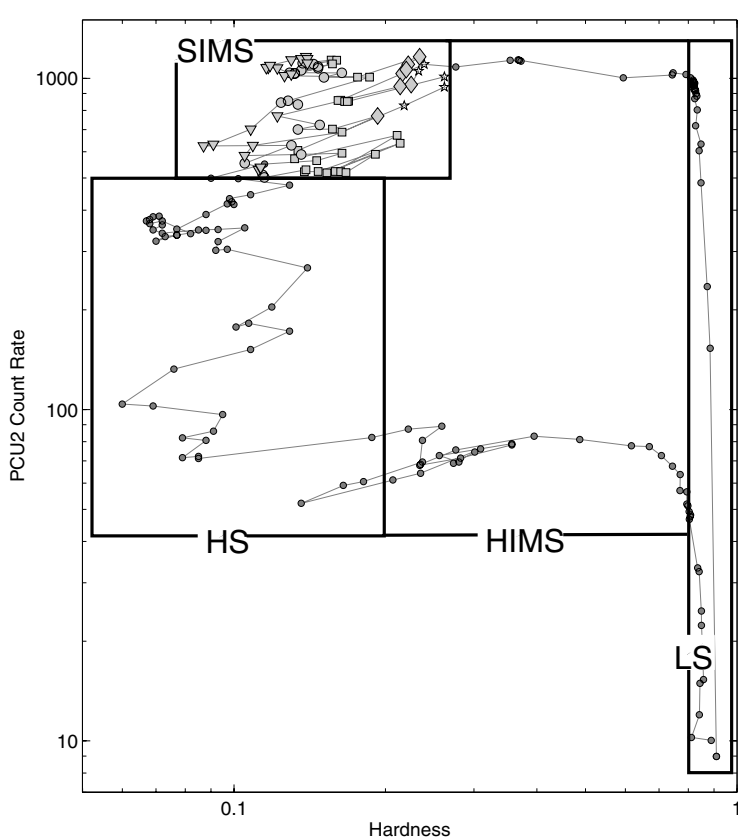

Fig. 18. Same Hardness-Intensity as in Fig. 2, but areas marking the regions corresponding to the source states described in the text.

type-C QPOs. No clear evidence of a SIMS is observed in these later stages of the outburst, although one observation shows a strong 1-Hz QPO which could be of type B. The HIMS evolves into a LS, roughly following an evolution opposite of that of the beginning of the outburst. These four states are observed in other systems and they are clearly separated by sharp transitions that are essential for their identification. Their location and boundaries on the HID of GX 339-4 are shown in Fig. 18.

The phenomenology of the states, their evolution and the transitions between them could constitute a firm starting point for the development of physical models for the outbursts of black-hole transients.

Acknowledgements. This work was partly supported by INAFPRIN 2002 grant and MIUR-PRIN grant 2003027534_004. We thank the anonymous referee for constructive comments.

\section{References}

Bailyn, C., \& Ferrara, L. 2004, ATel, 323, 1

Belloni, T. 2004, Proc. The Restless High-Energy Universe, Amsterdam, May 2003, ed. E. P. J. van den Heuvel, R. A. M. J. Wijers, \& J. M. M. in 't Zand, 337

Belloni, T., Parolin, I., \& Casella, P. 2004, A\&A, 423, 969

Belloni, T., van der Klis, M., Lewin, W. H. G., et al. 1997, A\&A, 322, 857

Belloni, T., Méndez, M., van der Klis, M., Lewin, W. H. G., \& Dieters, S., 1999, ApJ, 519, L159

Belloni, T., Psaltis, D., \& van der Klis, M. 2002, ApJ, 572, 392

Belloni, T., Homan, J., Cui, W., \& Swank, J. 2004, ATel, 236

Buxton, M., \& Bailyn, C. 2004, ATel, 270

Buxton, M., Gallo, E., Fender, R. P., \& Bailyn, C. 2004, ATel, 230

Casella, P., Belloni, T., Stella, L., \& Homan, J. 2004, A\&A, 426, 587
Corbel, S., Nowak, M. A., Fender, R. P., Tzioumis, A. H., \& Markoff, S. 2003, A\&A, 400, 1007

Corbel, S., Fender, R. P., Tomsick, J. A., Tzioumis, A. K., \& Tingay, S. 2004, ApJ, 617, 1272

Corongiu, A., Chiappetti, L., Haardt, F., Treves, A., Colpi, M., \& Belloni, T. 2003, A\&A, 408, 347

Cui, W., Zhang, S. N., Chen, W., \& Morgan, E. H. 1999, ApJ, 512, L43

Cui, W., Shrader, C. R., Haswell, C. A., \& Hynes, R. I. 2000, ApJ, 535, L123

Dieters, S., Belloni, T., Kuulkers, E., et al. 2000, ApJ, 538, 307

Fender, R. P., Corbel, S., Tzioumis, A. K., et al. 2002, ATel, 107

Fender R. P., \& Belloni, T. 2004, ARA\&A, 42, 317

Fender, R. P., Belloni, T., \& Gallo, E. 2004, MNRAS, 355, 1105

Gallo, E., Corbel, S., Fender, R. P., Maccarone, T. J., \& Tzioumis, A. K. 2004, MNRAS, 347, L52

Grebenev, S. A., Syunyaev, R. A., Pavlinskii, M. N., \& Dekhanov, I. A. 1999, Sov. Astr. Lett., 17, 413

Grove, J. E., Johnson, W. N., Kroeger, R. A., et al. 1998, ApJ, 500, 899

Homan, J., \& Belloni, T. 2005, Proc. workshop, From X-ray Binaries to Quasars: Black Hole Accretion on All Mass Scales, ed. T. Maccarone, \& R. P. Fender, in press [arXiv:astro-ph/0412597]

Homan, J., Wijnands, R., van der Klis, M., et al. 2001, ApJS, 132, 377

Homan, J., Klein-Wolt, M., Rossi, S., et al. 2003a, ApJ, 586, 1262

Homan, J., Miller, J. M., Wijnands, R., et al. 2003b, ATel, 161, 1

Homan, J. 2004, ATel, 318

Homan, J., Buxton, M., Markoff, S., et al. 2005a, ApJ, 624, 295

Homan, J., Miller, J. M., Wijnands, R., et al. 2005b, ApJ, 623, 383

Hynes, R. I., Steeghs, D., Casares, J., Charles, P. A., \& O'Brien, K. 2003, ApJ, 583, L95

Hynes, R. I., Steeghs, D., Casares, J., Charles, P. A., \& O’Brien, K. 2004, ApJ, 609, 317

Israel, G., Covino, S., Kuulkers, E., et al. 2004, ATel, 243

Kalemci, E., Tomsick, J. A., Rothschild, R. E., Pottschmidt, K., \& Kaaret, P. 2004, ApJ, 603, 231

Kanbach, G., Straubmeier, C., Spruit, H., \& Belloni, T. 2001, Nature, 414, 180

Klein-Wolt, M., Homan, J., \& van der Klis, M. 2004, Proc. The Restless High-Energy Universe, Amsterdam, May 2003, ed. E. P. J. van den Heuvel, R. A. M. J. Wijers, \& J. M. M. in 't Zand, 381

Kong, A. K. H., Kuulkers, E., Charles, P. A., \& Homer, L. 2000, MNRAS, 312, L49

Maejima, Y., Makishima, K., Matsuoka, M., et al. 1984, ApJ, 285, 712

Ilovaisky, S. A., Chevalier, C., Motch, C., \& Chiappetti, L. 1986, A\&A, 164, 67

McClintock, J. E., \& Remillard, R. A. 2005, in Compact Stellar X-Ray Sources, ed. W. H. G. Lewin, \& M. van der Klis, in press [arXiv: astro-ph/0306213]

Méndez, M., \& van der Klis, M. 1997, ApJ, 479, 926

Miller, J. M., Wijnands, R., Homan, J., et al. 2001, ApJ, 563, 928

Miller, J. M., Raymond, J., Fabian, A. C., et al. 2004a, ApJ, 601, 450

Miller, J. M., Fabian, A. C., Reynolds, C. S., et al. 2004b, ApJ, 606, L131

Miyamoto, S., Kimura, K., Kitamoto, S., Dotani, T., \& Ebisawa, K. 1991, ApJ, 383, 784

Miyamoto, S., Iga, S., Kitamoto, S., \& Kamado, Y. 1993, ApJ, 403, L39

Miyamoto, S., Kitamoto, S., Iga, S., Hayashida, K., \& Terada, K. 1994, ApJ, 435, 398

Morgan, E. H., Remillard, R. A., \& Greiner, J. 1997, ApJ, 482, 993

Nespoli, E., Belloni, T., Homan, J., et al. 2003, A\&A, 412, 235 
Nowak, M. 2000, MNRAS, 318, 361

Nowak, M. A., Wilms, J., \& Dove, J. B. 1999, ApJ, 517, 355

Nowak, M. A., Wilms, J., \& Dove, J. B. 2002, MNRAS, 332, 856

Olive, J. F., Barret, D., Boirin, L., et al. 1998, A\&A, 333, 942

Park, S. Q., Miller, J. M., McClintock, J. E., et al. 2004, ApJ, 610, 378

Pottschmidt, K., Wilms, J., Nowak, M. A., et al. 2000, A\&A, 357, L17

Reig, P., Belloni, T., van der Klis, M., et al. 2000, ApJ, 541, 883

Remillard, R. A., Morgan, E. H., McClintock, J. E., Bailyn, C. D., \& Orosz, J. A. 1999, ApJ, 522, 397

Remillard, R. A., Sobczak, G. J., Muno, M. P., \& McClintock, J. E. 2002, ApJ, 564, 962

Rodriguez, J., Corbel, S., Hannikainen, D. C., et al. 2004, ApJ, 615, 416

Smith, D. M., Swank, J. H., Heindl, W. A., \& Remillard, R. A. 2002a, ATel, 85

Smith, D. M., Belloni, T., Kalemci, E., et al. 2002b, IAUC, 7912

Smith, D. M., Belloni, T., Heindl, W. A., et al. 2002c, ATel, 95

Smith, D. M., Heindl, W. A., Swank, J. H., Wilms, J., \& Pottschmidt, K. 2004, ATel, 231

Sobczak, G. J., McClintock, J. E., Remillard, R. A., et al. 2000, ApJ, 544, 993

Strohmayer, T. E. 2001a, ApJ, 552, L49

Strohmayer, T. E. 2001b, ApJ, 554, L169

Takizawa, M., Dotani, T., Mitsuda, K., et al. 1997, ApJ, 489, 272
Tanaka, Y., \& Lewin, W. H. G. 1995, in X-ray Binaries, Cambridge Astrophysics Series (Cambridge, MA: Cambridge Univ. Press), ed. W. H. G. Lewin, J. van Paradijs, \& E. P. J. van den Heuvel, 126

Tomsick, J. A., Kalemci, E., \& Kaaret, P. 2004, ApJ, 601, 439

Trudolyubov, S. P., Borozdin, K. N., \& Priedhorsky, W. C. 2001, MNRAS, 322, 309

Trudolyubov, S. P. 2001, ApJ, 558, 276

van der Klis, M. 1995, in X-ray Binaries, Cambridge Astrophysics Series (Cambridge, MA: Cambridge Univ. Press), ed. W. H. G. Lewin, J. van Paradijs, \& E. P. J. van den Heuvel, 252

van der Klis, M. 2004, in Compact Stellar X-Ray Sources, ed. W. H. G. Lewin, \& M. van der Klis, in press [arXiv: astro-ph/0410551]

van Straaten, S., van der Klis, M., di Salvo, T., \& Belloni, T. 2002, ApJ, 568, 912

van Straaten, S., van der Klis, M., \& Méndez, M. 2003, ApJ, 596, 1155

Wijnands, R., Homan, J., \& van der Klis 1999, ApJ, 526, L33

Wilms, J., Nowak, M. A., Dove, J. B., Fender, R. P., \& Di Matteo, T. 1999, ApJ, 522, 460

Zdziarski, A. A., Grove, J. E., Poutanen, J., Rao, A. R., \& Vadawale, S. V. 2001, ApJ, 554, L45

Zdziarski, A. A., Gierliński, M., Mikołajewska, J., et al. 2004, MNRAS, 351, 791 
T. Belloni et al.: Timing GX 339-4 during its 2002/2003 outburst, Online Material p 1

\section{Online Material}


T. Belloni et al.: Timing GX 339-4 during its 2002/2003 outburst, Online Material p 2

Table 1. Log of the PCA observations. The headers mark the four source states (see text).

\begin{tabular}{|c|c|c|c|c|c|}
\hline $\begin{array}{l}\overline{O b s} \\
\#\end{array}$ & Obs. ID & $\overline{\mathrm{MJD}}$ & $\begin{array}{c}\text { Exp. } \\
\text { (s) }\end{array}$ & $\begin{array}{c}\text { PCU2 } \\
\mathrm{c} / \mathrm{s}\end{array}$ & 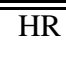 \\
\hline \multicolumn{6}{|c|}{ Low-hard state } \\
\hline 0 & $60705-01-54-00$ & 52359.64 & 768 & 9.0 & 0.91 \\
\hline 1 & $60705-01-55-00$ & 52366.60 & 896 & 153.2 & 0.88 \\
\hline 2 & 70109-01-02-00 & 52367.76 & 6784 & 235.3 & 0.87 \\
\hline 3 & 70109-01-01-00 & 52371.55 & 1408 & 483.7 & 0.85 \\
\hline 4 & 70110-01-01-00 & 52373.24 & 768 & 604.9 & 0.84 \\
\hline 5 & 70110-01-02-00 & 52374.42 & 512 & 633.1 & 0.85 \\
\hline 6 & 70109-01-03-00 & 52377.09 & 1280 & 719.1 & 0.83 \\
\hline 7 & 70110-01-03-00 & 52379.13 & 1536 & 802.5 & 0.83 \\
\hline 8 & 70110-01-04-00 & 52381.11 & 1536 & 868.4 & 0.82 \\
\hline 9 & 40031-03-01-00 & 52382.10 & 1792 & 882.9 & 0.83 \\
\hline 10 & $40031-03-02-00$ & 52383.09 & 2048 & 898.4 & 0.83 \\
\hline 11 & 40031-03-02-01 & 52384.12 & 3072 & 917.7 & 0.83 \\
\hline 12 & 40031-03-02-02 & 52384.93 & 4736 & 922.5 & 0.82 \\
\hline 13 & 70110-01-05-00 & 52385.46 & 768 & 925.3 & 0.83 \\
\hline 14 & 40031-03-02-03 & 52385.92 & 10496 & 945.9 & 0.82 \\
\hline 15 & 40031-03-02-04 & 52386.91 & 3584 & 952.9 & 0.82 \\
\hline 16 & 70110-01-06-00 & 52387.49 & 896 & 931.2 & 0.82 \\
\hline 17 & 70109-01-04-00 & 52387.56 & 10880 & 956.0 & 0.82 \\
\hline 18 & 40031-03-02-05 & 52388.06 & 1664 & 965.5 & 0.82 \\
\hline 19 & 40031-03-02-06 & 52389.06 & 1152 & 968.5 & 0.82 \\
\hline 20 & 70110-01-07-00 & 52390.91 & 896 & 977.6 & 0.82 \\
\hline 21 & 70109-01-05-01 & 52391.32 & 2432 & 978.3 & 0.82 \\
\hline 22 & $70109-01-05-00$ & 52391.38 & 3072 & 975.7 & 0.82 \\
\hline 23 & 70109-01-05-02 & 52391.45 & 2944 & 987.5 & 0.82 \\
\hline 24 & 70110-01-08-00 & 52394.44 & 1536 & 1003.3 & 0.81 \\
\hline \multicolumn{6}{|c|}{ Hard intermediate state } \\
\hline 25 & 70110-01-09-00 & 52398.66 & 1024 & 1027.3 & 0.79 \\
\hline 26 & 70109-01-06-00 & 52400.83 & 1920 & 1038.8 & 0.75 \\
\hline 27 & 70108-03-01-00 & 52400.85 & 8192 & 1023.4 & 0.74 \\
\hline 28 & 70110-01-10-00 & 52402.49 & 1024 & 1003.0 & 0.59 \\
\hline 29 & 70109-04-01-00 & 52405.58 & 5888 & 1136.7 & 0.37 \\
\hline 30 & 70109-04-01-01 & 52405.71 & 17408 & 1134.3 & 0.37 \\
\hline 31 & 70109-04-01-02 & 52406.07 & 1152 & 1127.3 & 0.37 \\
\hline 32 & 70110-01-11-00 & 52406.70 & 896 & 1132.3 & 0.35 \\
\hline 33 & 70110-01-12-00 & 52410.53 & 1152 & 1081.7 & 0.28 \\
\hline
\end{tabular}


T. Belloni et al.: Timing GX 339-4 during its 2002/2003 outburst, Online Material p 3

Table 1. continued.

\begin{tabular}{llcccc}
\hline \hline $\begin{array}{l}\text { Obs } \\
\#\end{array}$ & Obs. ID & MJD & $\begin{array}{c}\text { Exp. } \\
\text { (s) }\end{array}$ & $\begin{array}{c}\text { PCU2 } \\
\text { c/s }\end{array}$ & HR \\
\hline \multicolumn{6}{c}{ Soft intermediate state } \\
\hline 34 & $70109-01-07-00$ & 52411.60 & 5376 & 1102.5 & 0.24 \\
35 & $70110-01-13-00$ & 52412.07 & 896 & 1033.4 & 0.22 \\
36 & $40031-03-03-00$ & 52414.05 & 1024 & 858.9 & 0.16 \\
37 & $40031-03-03-01$ & 52414.12 & 640 & 857.0 & 0.16 \\
38 & $40031-03-03-02$ & 52414.19 & 384 & 850.8 & 0.17 \\
39 & $40031-03-03-03$ & 52414.29 & 1280 & 852.9 & 0.17 \\
40 & $40031-03-03-04$ & 52414.35 & 1664 & 851.1 & 0.17 \\
41 & $70110-01-14-00$ & 52416.60 & 768 & 1011.0 & 0.26 \\
42 & $70110-01-15-00$ & 52419.24 & 896 & 940.7 & 0.26 \\
43 & $70108-03-02-00$ & 52419.43 & 10368 & 827.2 & 0.22 \\
44 & $70110-01-16-00$ & 52423.16 & 640 & 605.9 & 0.13 \\
45 & $70109-01-08-00$ & 52424.34 & 7808 & 571.7 & 0.13 \\
46 & $70110-01-17-00$ & 52426.65 & 1024 & 672.8 & 0.21 \\
47 & $70110-01-18-00$ & 52430.65 & 896 & 523.1 & 0.16 \\
48 & $70109-01-09-00$ & 52431.78 & 2304 & 522.6 & 0.16 \\
49 & $70109-01-09-01$ & 52431.85 & 2048 & 519.4 & 0.17 \\
50 & $70109-01-09-02$ & 52431.92 & 1536 & 589.8 & 0.19 \\
51 & $70110-01-19-00$ & 52433.10 & 1152 & 518.4 & 0.15 \\
52 & $70110-01-20-00$ & 52437.44 & 512 & 636.6 & 0.21 \\
53 & $70109-01-10-00$ & 52438.45 & 4352 & 523.4 & 0.15 \\
54 & $70109-01-10-01$ & 52438.77 & 1024 & 522.2 & 0.14 \\
55 & $70109-01-10-02$ & 52438.84 & 1152 & 529.6 & 0.14 \\
56 & $70108-03-03-00$ & 52439.62 & 10752 & 502.1 & 0.12 \\
57 & $70110-01-21-00$ & 52440.51 & 512 & 530.4 & 0.11 \\
58 & $70109-03-01-00$ & 52440.75 & 12416 & 536.6 & 0.11 \\
59 & $70109-03-01-01$ & 52441.23 & 5888 & 510.5 & 0.11 \\
60 & $70109-03-01-02$ & 52441.84 & 6656 & 539.2 & 0.11 \\
\hline
\end{tabular}


Table 1. continued.

\begin{tabular}{|c|c|c|c|c|c|}
\hline $\begin{array}{l}\text { Obs } \\
\#\end{array}$ & Obs. ID & MJD & $\begin{array}{l}\text { Exp. } \\
\text { (s) }\end{array}$ & $\begin{array}{c}\text { PCU2 } \\
\mathrm{c} / \mathrm{s}\end{array}$ & $\overline{\mathrm{HR}}$ \\
\hline 61 & $70110-01-22-00$ & 52443.44 & 896 & 553.6 & 0.11 \\
\hline 62 & 70110-01-23-00 & 52448.34 & 768 & 627.5 & 0.13 \\
\hline 63 & $70109-01-12-00$ & 52451.58 & 3072 & 625.2 & 0.09 \\
\hline 64 & 70110-01-24-00 & 52451.89 & 896 & 631.9 & 0.09 \\
\hline 65 & $70110-01-25-00$ & 52454.03 & 768 & 701.7 & 0.11 \\
\hline 66 & 70110-01-26-00 & 52459.01 & 768 & 844.4 & 0.12 \\
\hline 67 & 70109-01-13-00 & 52459.56 & 8576 & 833.6 & 0.13 \\
\hline 68 & $70110-01-27-00$ & 52461.20 & 768 & 855.1 & 0.13 \\
\hline 69 & $70110-01-28-00$ & 52464.77 & 640 & 1008.4 & 0.19 \\
\hline 70 & $70109-01-15-00$ & 52468.31 & 10240 & 1018.6 & 0.13 \\
\hline 71 & 70110-01-29-00 & 52468.93 & 896 & 1030.0 & 0.13 \\
\hline 72 & $70110-01-30-00$ & 52472.95 & 896 & 1074.7 & 0.12 \\
\hline 73 & 70110-01-31-00 & 52475.00 & 768 & 1078.4 & 0.12 \\
\hline 74 & $70109-01-16-00$ & 52479.19 & 3584 & 1076.2 & 0.12 \\
\hline 75 & $70110-01-32-00$ & 52479.91 & 640 & 1092.8 & 0.12 \\
\hline 76 & 70110-01-33-00 & 52483.82 & 896 & 1163.2 & 0.14 \\
\hline 77 & 70110-01-34-00 & 52487.06 & 768 & 1136.6 & 0.13 \\
\hline 78 & 70109-01-17-00 & 52487.64 & 2688 & 1145.9 & 0.14 \\
\hline 79 & $70109-01-18-00$ & 52489.01 & 8832 & 1110.9 & 0.14 \\
\hline 80 & $70110-01-35-00$ & 52490.23 & 640 & 1134.8 & 0.16 \\
\hline 81 & $70110-01-36-00$ & 52494.11 & 896 & 1134.6 & 0.16 \\
\hline 82 & 70109-01-19-00 & 52495.28 & 7040 & 1105.7 & 0.14 \\
\hline 83 & $70110-01-37-00$ & 52496.09 & 896 & 1145.6 & 0.14 \\
\hline 84 & 70110-01-38-00 & 52499.97 & 1408 & 1111.5 & 0.14 \\
\hline 85 & 70109-01-20-00 & 52504.72 & 3328 & 1086.1 & 0.15 \\
\hline 86 & 70109-01-20-01 & 52504.78 & 4608 & 1056.2 & 0.14 \\
\hline 87 & 70110-01-40-00 & 52508.01 & 896 & 1105.1 & 0.16 \\
\hline 88 & $70109-01-21-00$ & 52509.59 & 3456 & 1032.5 & 0.13 \\
\hline $88 \mathrm{~b}$ & 70130-01-01-00 & 52510.45 & 3328 & 1071.3 & 0.15 \\
\hline 89 & $70110-01-41-00$ & 52511.05 & 896 & 1036.8 & 0.13 \\
\hline 90 & 70110-01-42-00 & 52514.08 & 896 & 1040.1 & 0.13 \\
\hline 91 & 70110-01-43-00 & 52517.86 & 640 & 1038.1 & 0.16 \\
\hline 92 & 70109-01-22-00 & 52518.23 & 3200 & 1007.2 & 0.15 \\
\hline 93 & 70110-01-44-00 & 52520.83 & 768 & 1008.0 & 0.18 \\
\hline 94 & $70110-01-45-00$ & 52524.95 & 1280 & 1161.3 & 0.23 \\
\hline 95 & 70110-01-46-00 & 52527.85 & 1024 & 1102.8 & 0.22 \\
\hline 96 & 70109-01-23-00 & 52529.58 & 1792 & 1062.7 & 0.22 \\
\hline 97 & 70110-01-47-00 & 52532.75 & 1280 & 1051.8 & 0.23 \\
\hline 98 & $70110-01-48-00$ & 52536.11 & 640 & 957.7 & 0.23 \\
\hline 99 & 70109-01-24-00 & 52536.36 & 2816 & 945.1 & 0.21 \\
\hline 100 & 70110-01-49-00 & 52538.49 & 896 & 769.5 & 0.12 \\
\hline 101 & $70110-01-50-00$ & 52542.53 & 896 & 722.8 & 0.15 \\
\hline 102 & $70109-01-25-00$ & 52543.23 & 4608 & 701.0 & 0.13 \\
\hline 103 & 70110-01-51-00 & 52545.85 & 640 & 702.7 & 0.16 \\
\hline $103 b$ & $70130-01-02-00$ & 52546.51 & 9344 & 769.9 & 0.19 \\
\hline 104 & 70109-01-11-00 & 52547.23 & 3840 & 687.9 & 0.16 \\
\hline 105 & $70110-01-52-00$ & 52550.20 & 896 & 627.1 & 0.11 \\
\hline 106 & $70110-01-53-00$ & 52553.11 & 896 & 584.2 & 0.11 \\
\hline 107 & 70109-01-14-00 & 52555.29 & 1664 & 589.1 & 0.14 \\
\hline 108 & $70110-01-54-00$ & 52555.80 & 1024 & 594.4 & 0.16 \\
\hline 109 & $70110-01-55-00$ & 52558.63 & 1024 & 564.4 & 0.15 \\
\hline
\end{tabular}


T. Belloni et al.: Timing GX 339-4 during its 2002/2003 outburst, Online Material p 5

Table 1. continued.

\begin{tabular}{|c|c|c|c|c|c|}
\hline $\begin{array}{l}\text { Obs } \\
\#\end{array}$ & Obs. ID & MJD & $\begin{array}{l}\text { Exp. } \\
\text { (s) }\end{array}$ & $\begin{array}{c}\text { PCU2 } \\
\mathrm{c} / \mathrm{s}\end{array}$ & $\overline{\mathrm{HR}}$ \\
\hline \multicolumn{6}{|c|}{ High/soft sate } \\
\hline 110 & 70109-01-26-00 & 52560.41 & 3200 & 550.7 & 0.12 \\
\hline 111 & $70110-01-56-00$ & 52562.92 & 896 & 499.9 & 0.09 \\
\hline 112 & 70110-01-57-00 & 52565.81 & 1024 & 497.3 & 0.10 \\
\hline 113 & 70110-01-58-00 & 52569.73 & 640 & 476.0 & 0.13 \\
\hline 114 & 70110-01-59-00 & 52574.32 & 896 & 445.4 & 0.11 \\
\hline 115 & 70110-01-60-00 & 52577.81 & 768 & 433.6 & 0.10 \\
\hline 116 & 70110-01-61-00 & 52579.67 & 896 & 423.6 & 0.10 \\
\hline 117 & 70109-01-11-02 & 52579.77 & 1280 & 416.4 & 0.10 \\
\hline 118 & 70109-01-27-00 & 52579.84 & 1664 & 418.2 & 0.10 \\
\hline 119 & 70110-01-62-00 & 52584.40 & 768 & 388.1 & 0.09 \\
\hline 120 & 70110-01-63-00 & 52588.49 & 768 & 350.7 & 0.08 \\
\hline 121 & 70110-01-64-00 & 52591.95 & 896 & 336.2 & 0.08 \\
\hline 122 & 70110-01-65-00 & 52595.29 & 1280 & 332.8 & 0.07 \\
\hline 123 & 70109-01-28-00 & 52595.77 & 4096 & 322.5 & 0.07 \\
\hline 124 & $70110-01-6$ & 52597.33 & 1024 & 336.4 & 0.08 \\
\hline 125 & 70110-01-67-00 & 52600.79 & 640 & 347.8 & 0.09 \\
\hline 126 & 70110-01-68-00 & 52603.70 & 768 & 339.9 & 0.08 \\
\hline 127 & 70110-01-69-00 & 52635.71 & 896 & 382.0 & 0.07 \\
\hline 128 & 70109-01- & 52636.74 & 2432 & 383.5 & 0.07 \\
\hline 129 & 70110-01-70-00 & 52640.31 & 896 & 375.0 & 0.07 \\
\hline 130 & 70110-01-71-00 & 52642.14 & 896 & 371.0 & 0.07 \\
\hline 131 & 70109-01-30-00 & 52647.08 & 3840 & 364.1 & 0.07 \\
\hline 132 & 70110-01-72-00 & 52648.27 & 768 & 371.2 & 0.07 \\
\hline 133 & 70110-01- & 52650.14 & 1024 & 360.8 & 0.07 \\
\hline 134 & 70109-01-31-00 & 52652.15 & 4096 & 348.9 & 0.07 \\
\hline 135 & 70110-01-74-00 & 52654.59 & 768 & 340.9 & 0.07 \\
\hline 136 & 70110-01-75-00 & 52657.12 & 896 & 348.6 & 0.09 \\
\hline 137 & 70109-01-32-00 & 52658.93 & 2944 & 350.0 & 0.09 \\
\hline 138 & 70110-01-76-00 & 52660.64 & 896 & 353.4 & 0.10 \\
\hline 139 & 70110-01-77-00 & 52663.47 & 768 & 321.4 & 0.09 \\
\hline 140 & 70109-01-33-00 & 52667.02 & 3456 & 302.6 & 0.09 \\
\hline 141 & 70110-01- & 52667.45 & 640 & 304.8 & 0.10 \\
\hline 142 & 70110-01-79-00 & 52671.26 & 768 & 267.7 & 0.14 \\
\hline $142 b$ & 70110-01-80-00 & 52675.65 & 896 & 204.1 & 0.12 \\
\hline $143 b$ & 70109-01-34-01G & 52676.64 & 1024 & 177.5 & 0.10 \\
\hline 143 & 70109-01-34-00 & 52676.95 & 2944 & 182.1 & 0.11 \\
\hline $143 c$ & 70110-01-81-00 & 52678.96 & 896 & 172.5 & 0.13 \\
\hline 144 & 70109-01-35-01 & 52680.20 & 1536 & 152.0 & 0.11 \\
\hline 145 & 70109-01-35-00 & 52680.84 & 2816 & 132.5 & 0.08 \\
\hline $145 b$ & 70110-01-82-00 & 52683.50 & 896 & 104.0 & 0.06 \\
\hline 146 & 70109-01-36-01 & 52684.35 & 1024 & 102.5 & 0.07 \\
\hline $146 b$ & 70110-01-83-00 & 52686.25 & 896 & 96.6 & 0.10 \\
\hline 147 & 70109-01-36-00 & 52687.69 & 2432 & 86.1 & 0.09 \\
\hline 148 & 70109-01-36-02 & 52688.29 & 1152 & 82.2 & 0.08 \\
\hline $148 b$ & 70110-01-84-00 & 52688.89 & 640 & 80.7 & 0.09 \\
\hline $148 \mathrm{c}$ & 70111-01-01-000 & 52690.57 & 15872 & 71.6 & 0.08 \\
\hline $148 d$ & 70111-01-01-00 & 52690.84 & 3328 & 72.3 & 0.09 \\
\hline $148 \mathrm{e}$ & 70128-02-01-01 & 52690.98 & 1792 & 71.2 & 0.08 \\
\hline $148 \mathrm{f}$ & 70110-01-86-00 & 52693.73 & 896 & 82.3 & 0.19 \\
\hline
\end{tabular}


Table 1. continued.

\begin{tabular}{|c|c|c|c|c|c|}
\hline $\begin{array}{l}\text { Obs } \\
\#\end{array}$ & Obs. ID & MJD & $\begin{array}{c}\text { Exp. } \\
\text { (s) }\end{array}$ & $\begin{array}{c}\text { PCU2 } \\
\mathrm{c} / \mathrm{s}\end{array}$ & HR \\
\hline \multicolumn{6}{|c|}{ Hard intermediate state } \\
\hline 149 & 70109-01-37-00 & 52694.92 & 3712 & 87.2 & 0.22 \\
\hline $149 b$ & 70128-02-02-00 & 52696.36 & 20608 & 89.1 & 0.26 \\
\hline $149 \mathrm{c}$ & 70110-01-85-00 & 52697.14 & 1024 & 80.8 & 0.24 \\
\hline $149 d$ & 70110-01-87-00 & 52700.60 & 1152 & 67.9 & 0.23 \\
\hline $149 \mathrm{e}$ & 70110-01-88-00 & 52704.02 & 768 & 71.4 & 0.28 \\
\hline $149 f$ & 50117-01-03-01 & 52706.77 & 2816 & 78.3 & 0.36 \\
\hline $149 \mathrm{~g}$ & $50117-01-03-00$ & 52706.84 & 7808 & 78.9 & 0.36 \\
\hline $149 \mathrm{~h}$ & 70110-01-89-00 & 52707.92 & 896 & 72.7 & 0.26 \\
\hline 151 & 70109-02-01-00 & 52709.86 & 6912 & 69.4 & 0.28 \\
\hline 150 & 70109-02-01-01 & 52709.99 & 3584 & 68.8 & 0.27 \\
\hline 152 & $60705-01-56-00$ & 52710.72 & 2560 & 74.4 & 0.30 \\
\hline $152 b$ & 70110-01-90-00 & 52711.51 & 1024 & 76.1 & 0.31 \\
\hline 153 & 70109-02-02-00 & 52713.08 & 6912 & 64.3 & 0.24 \\
\hline $153 b$ & 70110-01-91-00 & 52713.48 & 896 & 61.3 & 0.21 \\
\hline $153 \mathrm{c}$ & 50117-01-04-00 & 52715.85 & 17408 & 52.1 & 0.14 \\
\hline 154 & $60705-01-57-00$ & 52716.70 & 2560 & 59.0 & 0.16 \\
\hline $154 \mathrm{~b}$ & 70110-01-92-00 & 52717.50 & 1024 & 60.6 & 0.18 \\
\hline $154 \mathrm{c}$ & 50117-01-04-01 & 52718.08 & 3456 & 69.5 & 0.24 \\
\hline $154 \mathrm{c} 2$ & 50117-01-04-02 & 52718.17 & 1280 & 68.3 & 0.24 \\
\hline $154 d$ & 70110-01-93-00 & 52719.23 & 1024 & 75.5 & 0.28 \\
\hline $154 \mathrm{e}$ & 70110-01-94-00 & 52724.23 & 896 & 83.1 & 0.40 \\
\hline $154 \mathrm{f}$ & 70110-01-95-00 & 52727.25 & 896 & 81.3 & 0.49 \\
\hline 155 & $60705-01-59-00$ & 52731.56 & 3456 & 77.7 & 0.62 \\
\hline $155 b$ & 70110-01-96-00 & 52732.11 & 896 & 77.3 & 0.67 \\
\hline $155 \mathrm{c}$ & 70110-01-97-00 & 52734.22 & 1152 & 72.7 & 0.71 \\
\hline 156 & 80116-02-01-00 & 52735.64 & 3584 & 67.4 & 0.74 \\
\hline $156 b$ & 70110-01-98-00 & 52737.25 & 1152 & 63.7 & 0.77 \\
\hline 157 & 80116-02-01-02 & 52739.58 & 3840 & 57.0 & 0.77 \\
\hline $157 b$ & 70110-01-01-10 & 52740.01 & 896 & 56.4 & 0.79 \\
\hline \multicolumn{6}{|c|}{ Low/Hard state } \\
\hline 158 & $80116-02-02-00$ & 52741.70 & 6016 & 51.8 & 0.79 \\
\hline 159 & 80116-02-02-01 & 52742.04 & 2176 & 51.2 & 0.80 \\
\hline $158 b$ & 70128-02-03-00 & 52742.24 & 14464 & 49.2 & 0.80 \\
\hline $159 b$ & 70128-02-03-01 & 52742.61 & 10496 & 48.0 & 0.81 \\
\hline $159 \mathrm{c}$ & 70110-01-02-10 & 52743.16 & 768 & 47.5 & 0.81 \\
\hline 160 & $80116-02-02-02$ & 52743.27 & 8448 & 46.7 & 0.80 \\
\hline 161 & 60705-01-61-00 & 52746.97 & 2816 & 33.3 & 0.84 \\
\hline $161 b$ & 70110-01-03-10 & 52747.40 & 1536 & 32.5 & 0.84 \\
\hline 162 & $60705-01-62-00$ & 52750.36 & 3456 & 24.7 & 0.85 \\
\hline $162 b$ & 70110-01-04-10 & 52751.57 & 768 & 22.3 & 0.85 \\
\hline 163 & 60705-01-63-00 & 52756.04 & 3840 & 15.3 & 0.86 \\
\hline $162 \mathrm{c}$ & 70110-01-05-10 & 52756.38 & 768 & 14.9 & 0.84 \\
\hline $163 b$ & 70110-01-06-10 & 52760.21 & 768 & 12.0 & 0.84 \\
\hline $163 \mathrm{c}$ & 80102-04-01-00 & 52763.34 & 896 & 10.2 & 0.81 \\
\hline 164 & 60705-01-64-00 & 52765.64 & 3072 & 10.0 & 0.89 \\
\hline
\end{tabular}


T. Belloni et al.: Timing GX 339-4 during its 2002/2003 outburst, Online Material p 7

Table 2. Main characteristic frequencies (in $\mathrm{Hz}$ ) for the right and top branches of the HID.

\begin{tabular}{|c|c|c|c|c|c|c|}
\hline Obs \# & $v_{0}$ & $v_{1}$ & $v_{Q}$ & $v_{2}$ & $v_{3}$ & $v_{i}$ \\
\hline \multicolumn{7}{|c|}{ Right branch } \\
\hline 1 & $\ldots$ & $0.012 \pm 0.005$ & $\ldots$ & $2.23 \pm 0.20$ & $\ldots$ & $0.28 \pm 0.06$ \\
\hline 2 & $\ldots$ & $0.03 \pm 0.01$ & $\ldots$ & $2.22 \pm 0.05$ & $\ldots$ & $0.38 \pm 0.04$ \\
\hline 3 & $\ldots$ & $0.06 \pm 0.01$ & $\ldots$ & $2.52 \pm 0.07$ & $\ldots$ & $\ldots$ \\
\hline 4 & $\ldots$ & $0.07 \pm 0.01$ & $\ldots$ & $2.41 \pm 0.07$ & $\ldots$ & $\ldots$ \\
\hline 5 & $\ldots$ & $0.06 \pm 0.01$ & $\ldots$ & $2.34 \pm 0.08$ & $\ldots$ & $\ldots$ \\
\hline 6 & $\ldots$ & $0.08 \pm 0.01$ & $\ldots$ & $2.88 \pm 0.21$ & $\ldots$ & $\ldots$ \\
\hline 7 & $\ldots$ & $0.13 \pm 0.01$ & $\ldots$ & $3.21 \pm 0.05$ & $\ldots$ & $\ldots$ \\
\hline 8 & $\ldots$ & $0.12 \pm 0.01$ & $\ldots$ & $3.09 \pm 0.07$ & $24.14 \pm 1.85$ & $\ldots$ \\
\hline 9 & $\ldots$ & $0.14 \pm 0.01$ & $\ldots$ & $3.06 \pm 0.09$ & $24.85 \pm 2.82$ & $\ldots$ \\
\hline 10 & $\ldots$ & $0.15 \pm 0.01$ & $\ldots$ & $3.08 \pm 0.08$ & $\ldots$ & $\ldots$ \\
\hline 11 & $\ldots$ & $0.14 \pm 0.01$ & $\ldots$ & $2.85 \pm 0.05$ & $\ldots$ & $\ldots$ \\
\hline 12 & $\ldots$ & $0.14 \pm 0.05$ & $\ldots$ & $2.99 \pm 0.04$ & $31.92 \pm 2.28$ & $\ldots$ \\
\hline 13 & $\ldots$ & $0.13 \pm 0.01$ & $\ldots$ & $3.06 \pm 0.16$ & $\ldots$ & $\ldots$ \\
\hline 14 & $\ldots$ & $0.15 \pm 0.03$ & $\ldots$ & $3.21 \pm 0.03$ & $29.38 \pm 1.61$ & $\ldots$ \\
\hline 15 & $\ldots$ & $0.17 \pm 0.01$ & $\ldots$ & $3.33 \pm 0.05$ & $34.40 \pm 4.23$ & $\ldots$ \\
\hline 16 & $\ldots$ & $0.20 \pm 0.02$ & $\ldots$ & $3.49 \pm 0.12$ & $30.32 \pm 8.30$ & $\ldots$ \\
\hline 17 & $\ldots$ & $0.17 \pm 0.01$ & $0.16 \pm 0.01$ & $3.26 \pm 0.03$ & $28.38 \pm 0.97$ & $\ldots$ \\
\hline 18 & $\ldots$ & $0.20 \pm 0.01$ & $0.20 \pm 0.01$ & $3.54 \pm 0.08$ & $28.87 \pm 3.66$ & $\ldots$ \\
\hline 19 & $\ldots$ & $0.16 \pm 0.01$ & $\ldots$ & $3.33 \pm 0.08$ & $33.34 \pm 3.26$ & $\ldots$ \\
\hline 20 & $\ldots$ & $0.18 \pm 0.01$ & $\ldots$ & $3.06 \pm 0.11$ & $\ldots$ & $\ldots$ \\
\hline 21 & $\ldots$ & $0.23 \pm 0.01$ & $0.20 \pm 0.01$ & $3.71 \pm 0.07$ & $36.48 \pm 2.74$ & $\ldots$ \\
\hline 22 & $\ldots$ & $0.22 \pm 0.01$ & $\ldots$ & $3.39 \pm 0.10$ & $30.08 \pm 4.60$ & $\ldots$ \\
\hline 23 & $\ldots$ & $0.22 \pm 0.01$ & $\ldots$ & $3.81 \pm 0.14$ & $26.15 \pm 2.68$ & $\ldots$ \\
\hline 24 & $\ldots$ & $0.26 \pm 0.02$ & $\ldots$ & $3.72 \pm 0.08$ & $38.68 \pm 3.14$ & $\ldots$ \\
\hline 25 & $\ldots$ & $0.55 \pm 0.05$ & $\ldots$ & $4.88 \pm 0.16$ & $35.20 \pm 2.81$ & $\ldots$ \\
\hline \multicolumn{7}{|c|}{ Top branch } \\
\hline 26 & $\ldots$ & $1.21 \pm 0.03$ & $1.26 \pm 0.01$ & $9.59 \pm 0.23$ & $39.31 \pm 1.64$ & $\ldots$ \\
\hline 27 & $\ldots$ & $1.23 \pm 0.02$ & $1.33 \pm 0.01$ & $9.63 \pm 0.31$ & $43.65 \pm 1.85$ & $\ldots$ \\
\hline 28 & $\ldots$ & $3.31 \pm 0.12$ & $2.08 \pm 0.01$ & $\ldots$ & $49.33 \pm 6.03$ & $\ldots$ \\
\hline 29 & $1.08 \pm 0.01$ & $6.46 \pm 0.26$ & $5.46 \pm 0.01$ & $\ldots$ & $\ldots$ & $\ldots$ \\
\hline 30 & $0.99 \pm 0.01$ & $5.93 \pm 0.13$ & $5.45 \pm 0.01$ & $\ldots$ & $96.93 \pm 14.78$ & $\ldots$ \\
\hline 31 & $0.94 \pm 0.02$ & $5.73 \pm 0.61$ & $5.34 \pm 0.02$ & $\ldots$ & $\ldots$ & $\ldots$ \\
\hline 32 & $0.99 \pm 0.01$ & $6.08 \pm 0.75$ & $5.82 \pm 0.02$ & $\ldots$ & $\ldots$ & $\ldots$ \\
\hline 33 & $0.99 \pm 0.01$ & $\ldots$ & $8.12 \pm 0.18$ & $\ldots$ & $\ldots$ & $\ldots$ \\
\hline
\end{tabular}


T. Belloni et al.: Timing GX 339-4 during its 2002/2003 outburst, Online Material p 8

Table 4. Main characteristic frequencies (in $\mathrm{Hz}$ ) for the bottom branch of the HID.

\begin{tabular}{|c|c|c|c|c|}
\hline Obs \# & $v_{1}$ & $v_{2}$ & $v_{3}$ & $v_{Q}$ \\
\hline \multicolumn{5}{|c|}{ Bottom branch } \\
\hline 149 & $2.91 \pm 0.21$ & $\ldots$ & $\ldots$ & $8.59 \pm 0.18$ \\
\hline $149 b$ & $1.87 \pm 0.10$ & $5.42 \pm 0.80$ & $\ldots$ & $8.02 \pm 0.04$ \\
\hline $149 \mathrm{c}$ & $2.58 \pm 0.19$ & $\ldots$ & $\ldots$ & $\ldots$ \\
\hline $149 d$ & $3.24 \pm 0.44$ & $\ldots$ & $\ldots$ & $\ldots$ \\
\hline $149 \mathrm{e}$ & $3.13 \pm 0.28$ & $\ldots$ & $\ldots$ & $\ldots$ \\
\hline $149 f$ & $3.19 \pm 0.49$ & $\ldots$ & $\ldots$ & $6.72 \pm 0.06$ \\
\hline $149 \mathrm{~g}$ & $3.39 \pm 0.22$ & $\ldots$ & $\ldots$ & $6.77 \pm 0.02$ \\
\hline $149 \mathrm{~h}$ & $\ldots$ & $\ldots$ & $\ldots$ & $\ldots$ \\
\hline 151 & $2.71 \pm 0.11$ & $\ldots$ & $\ldots$ & $8.03 \pm 0.06$ \\
\hline 150 & $3.04 \pm 0.24$ & $\ldots$ & $\ldots$ & $8.10 \pm 0.13$ \\
\hline 152 & $4.00 \pm 0.57$ & $\ldots$ & $\ldots$ & $7.77 \pm 0.09$ \\
\hline $152 b$ & $2.82 \pm 0.37$ & $\ldots$ & $\ldots$ & $\ldots$ \\
\hline 153 & $3.33 \pm 0.21$ & $\ldots$ & $\ldots$ & $\ldots$ \\
\hline $153 b$ & $3.84 \pm 0.56$ & $\ldots$ & $\ldots$ & $\ldots$ \\
\hline $153 \mathrm{c}$ & $\ldots$ & $\ldots$ & $\ldots$ & $\ldots$ \\
\hline 154 & $\ldots$ & $\ldots$ & $\ldots$ & $\ldots$ \\
\hline $154 b$ & $\ldots$ & $\ldots$ & $\ldots$ & $\ldots$ \\
\hline $154 \mathrm{c}$ & $3.37 \pm 0.29$ & $\ldots$ & $\ldots$ & $\ldots$ \\
\hline $154 \mathrm{~g}$ & $3.01 \pm 0.26$ & $\ldots$ & $\ldots$ & $\ldots$ \\
\hline $154 d$ & $3.13 \pm 0.53$ & $\ldots$ & $\ldots$ & $\ldots$ \\
\hline $154 \mathrm{e}$ & $3.81 \pm 0.49$ & $\ldots$ & $\ldots$ & $6.12 \pm 0.10$ \\
\hline $154 f$ & $1.09 \pm 0.09$ & $6.64 \pm 1.20$ & $\ldots$ & $4.74 \pm 0.07$ \\
\hline 155 & $0.67 \pm 0.06$ & $4.22 \pm 0.86$ & $\ldots$ & $2.85 \pm 0.07$ \\
\hline $155 b$ & $0.44 \pm 0.08$ & $2.86 \pm 0.48$ & $\ldots$ & $\ldots$ \\
\hline $155 \mathrm{c}$ & $0.48 \pm 0.21$ & $2.53 \pm 0.87$ & $\ldots$ & $\ldots$ \\
\hline 156 & $0.22 \pm 0.02$ & $1.90 \pm 0.10$ & $\ldots$ & $\ldots$ \\
\hline $156 b$ & $0.24 \pm 0.03$ & $1.45 \pm 0.20$ & $\ldots$ & $\ldots$ \\
\hline 157 & $0.18 \pm 0.03$ & $1.48 \pm 0.08$ & $\ldots$ & $\ldots$ \\
\hline $157 b$ & $0.27 \pm 0.03$ & $1.90 \pm 0.44$ & $\ldots$ & $\ldots$ \\
\hline 158 & $0.20 \pm 0.05$ & $1.33 \pm 0.19$ & $\ldots$ & $\ldots$ \\
\hline 159 & $0.22 \pm 0.02$ & $1.15 \pm 0.08$ & $\ldots$ & $\ldots$ \\
\hline $158 b$ & $0.28 \pm 0.02$ & $1.22 \pm 0.05$ & $6.13 \pm 0.56$ & $\ldots$ \\
\hline $159 b$ & $0.23 \pm 0.02$ & $1.17 \pm 0.06$ & $6.31 \pm 0.69$ & $\ldots$ \\
\hline $159 \mathrm{c}$ & $0.14 \pm 0.05$ & $1.25 \pm 0.02$ & $\ldots$ & $\ldots$ \\
\hline 160 & $0.36 \pm 0.06$ & $2.44 \pm 0.24$ & $\ldots$ & $\ldots$ \\
\hline 161 & $0.14 \pm 0.03$ & $1.40 \pm 0.39$ & $\ldots$ & $\ldots$ \\
\hline $161 b$ & $0.13 \pm 0.04$ & $0.85 \pm 0.21$ & $\ldots$ & $\ldots$ \\
\hline 162 & $0.12 \pm 0.03$ & $2.11 \pm 0.58$ & $\ldots$ & $\ldots$ \\
\hline $162 b$ & $0.23 \pm 0.05$ & $2.92 \pm 1.29$ & $\ldots$ & $\ldots$ \\
\hline 163 & $0.11 \pm 0.01$ & $1.84 \pm 0.32$ & $\ldots$ & $\ldots$ \\
\hline $162 \mathrm{c}$ & $0.18 \pm 0.03$ & $3.17 \pm 0.18$ & $\ldots$ & $\ldots$ \\
\hline $163 b$ & $0.13 \pm 0.03$ & $1.21 \pm 0.04$ & $\ldots$ & $\ldots$ \\
\hline $163 c$ & $0.23 \pm 0.06$ & $1.85 \pm 0.62$ & $\ldots$ & $\ldots$ \\
\hline 164 & $0.06 \pm 0.01$ & $1.33 \pm 0.31$ & $\ldots$ & $\ldots$ \\
\hline
\end{tabular}




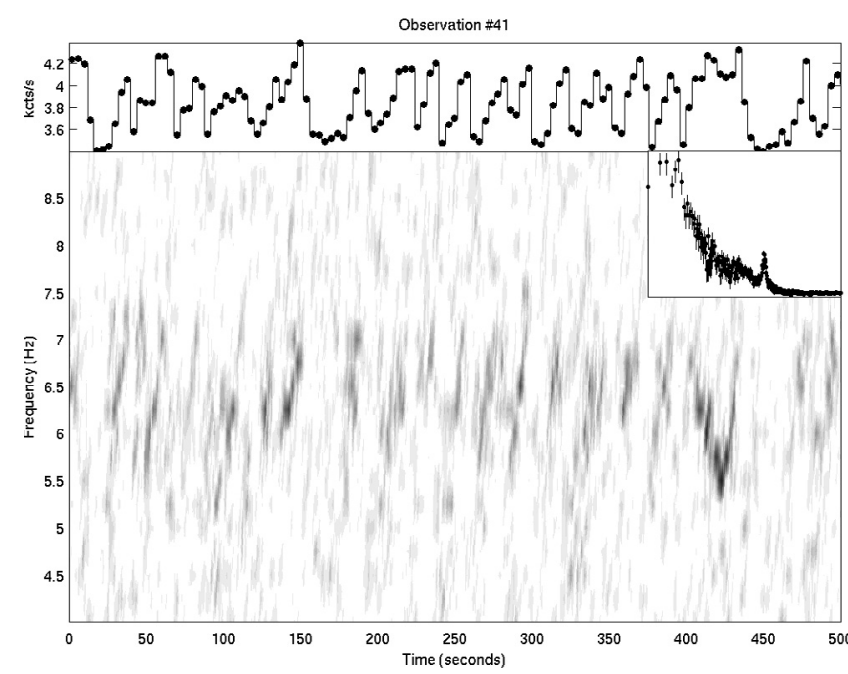

Fig. A.1. Same as Fig. 9 for Obs. \#41.

\section{Appendix A: Type-B QPOs in detail}

In this appendix, we describe the detailed analysis of the four type-B observations found in addition to that reported by Nespoli et al. (2003). Notice that the fits to average power spectra described below are only indicative, since it is clear that there are major fast variations in the QPO parameters on rather short time scales (see also Nespoli et al. 2003).

- Observation \#41: from Fig. A.1, it is evident that the light curve (top panel) is characterized by oscillations between two flux levels. From the corresponding spectrogram (bottom panel) one can see that the QPO is present only during the high-flux intervals, where it is correlated with count rate. From the spectrogram, we produced two average power spectra by selecting all power spectra corresponding to a count rate $<3400 \mathrm{cts} / \mathrm{s}$ and $>3500 \mathrm{cts} / \mathrm{s}$ respectively (3 PCUs were on during this observation). In the power spectrum corresponding to the low count-rate intervals (see Fig. A.1), no QPO is indeed visible: a fit with a zerocentered Lorentzian yields a characteristic frequency $v_{b}=$ $2.68 \pm 0.32 \mathrm{~Hz}$ and a fractional $\mathrm{rms}$ of $\sim 8 \%$. In the power spectrum corresponding to high count rates, a narrow QPO is visible. A Gaussian fit yields $v_{q}=6.45 \pm 0.03 \mathrm{~Hz}$ and $\sigma=0.52 \pm 0.03 \mathrm{~Hz}$, with an integrated fractional rms of $\sim 5 \%$. A band-limited noise component is also seen: a Lorentzian fit gives $v_{b}=5.74 \pm 0.44 \mathrm{~Hz}$ and a fractional rms of $\sim 6.5 \%$. Additional power is present at low frequencies $(<1 \mathrm{~Hz})$. The integrated $0.1-64 \mathrm{~Hz}$ fractional $\mathrm{rms}$ is similar between the low and high count rate power spectra.

- Observation \#42: here the situation is slightly different. The QPO is only visible in the high-flux intervals, as for Obs. \#41, but the low-flux intervals look more like dips in the light curve as they are relatively few and sparse. Again, we extracted two average power spectra from the 4s spectrogram (see Fig. A.2). During the dips (rate $<4200$ cts/s for 4 PCUs), no significant QPO is visible: a good fit is obtained with a broad Lorentzian component with $v_{\max }=4.23 \pm 0.39 \mathrm{~Hz}$, plus an excess at low frequencies. Outside the dips, the narrow QPO appears at an av-

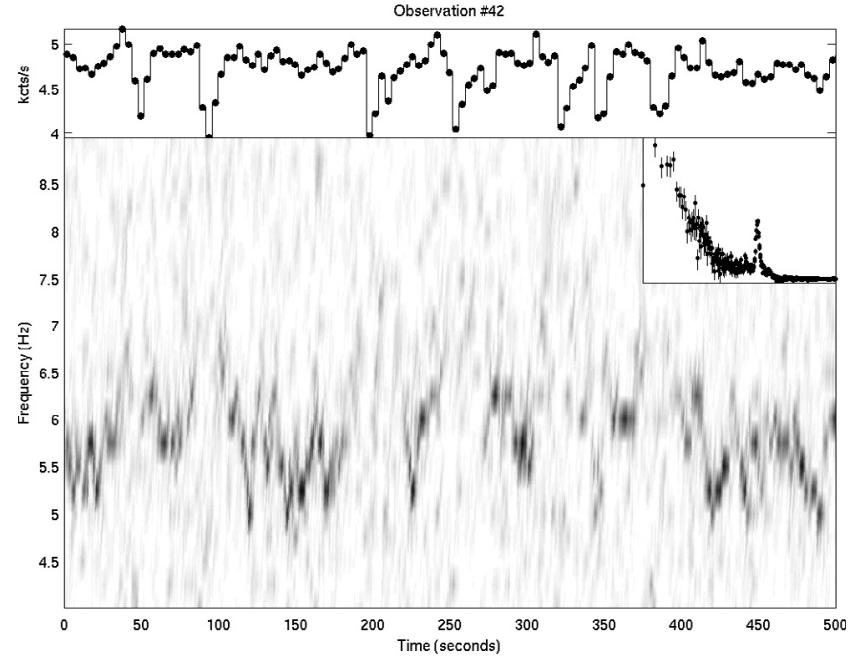

Fig. A.2. Same as Fig. A.1 for Obs. \#42.

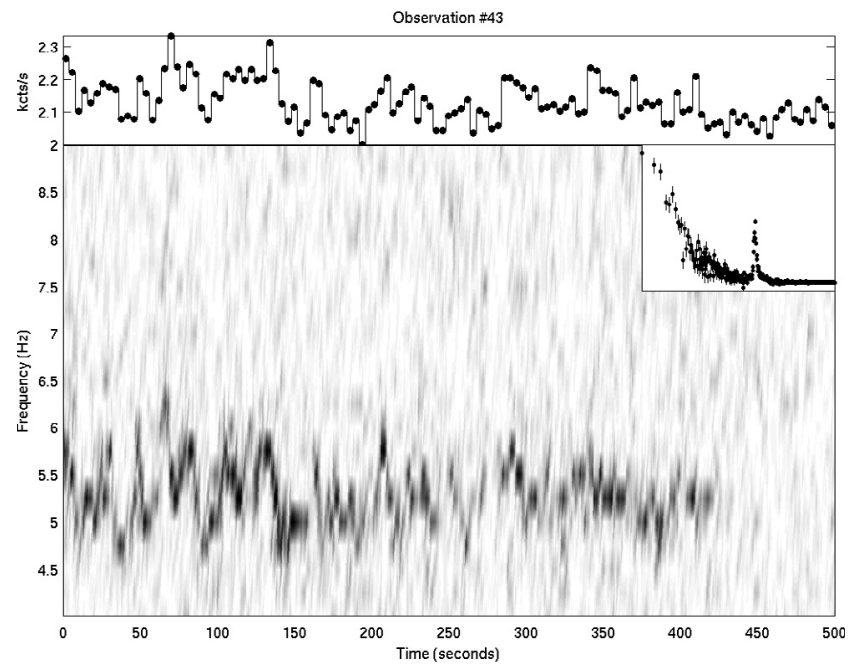

Fig. A.3. Same as Fig. A.1 for Obs. \#43.

erage frequency of $v_{q}=5.79 \pm 0.02 \mathrm{~Hz}$ (Gaussian fit) with $\sigma=0.39 \pm 0.02 \mathrm{~Hz}$ and with an integrated fractional rms of $\sim 5.6 \%$. A subharmonic and a second harmonic components are also evident. The continuum noise can be approximated with a broad Lorentzian component $\left(v_{\max }=6.62 \pm 0.34 \mathrm{~Hz}\right)$, with as usual some excess at low frequencies. As for Obs. \#41, the integrated $0.1-64 \mathrm{~Hz}$ fractional rms is similar between the low and high count rate power spectra.

- Observation \#43: from Fig. A.3 the presence of a similar narrow QPO peak is evident. The observation consists of three separate segments of 23400 s exposure each separated by 2200 s-long gaps. The QPO is present only in the first $\sim 1550 \mathrm{~s}$ of the second interval, after which it disappears on a time scale of a few seconds (see Fig. A.3). The QPO is clearly related to additional low-frequency noise in the light curve (see Fig. A.3). Accumulating the $128 \mathrm{~s}$ power spectrum corresponding to the interval when the QPO is present (in this case the 4 s resolution is not needed), a clear peak is present. We fitted it with a Gaussian with centroid frequency $v_{q}=5.35 \pm 0.01 \mathrm{~Hz}$ and $\sigma=$ 


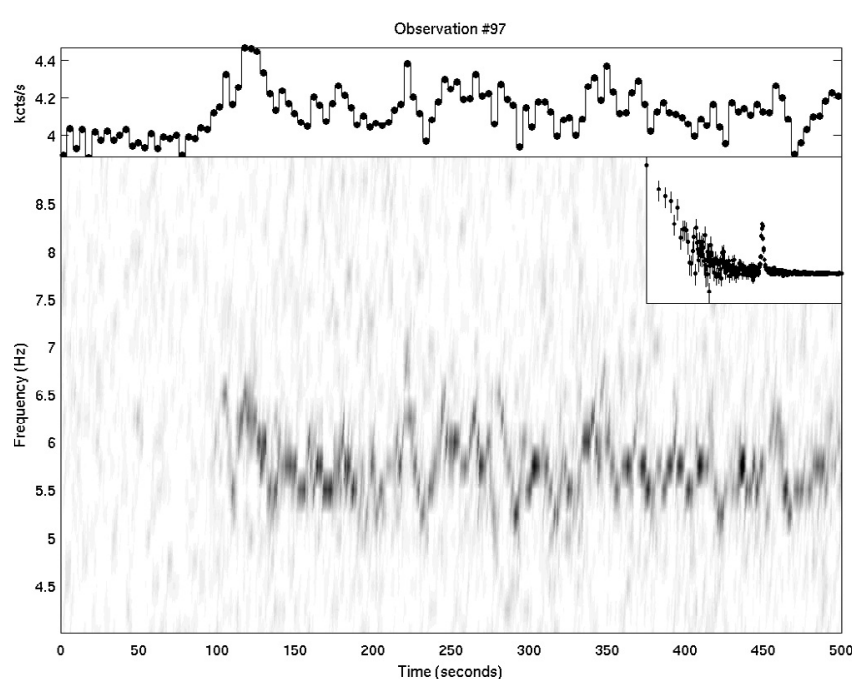

Fig. A.4. Same as Fig. A.1 for Obs. \#97.

$0.38 \pm 0.01 \mathrm{~Hz}$, for an integrated fractional rms of $7.7 \%$. The continuum noise can be fitted with two zero-centered Lorentzians with characteristic frequencies $0.02 \pm 0.01 \mathrm{~Hz}$ and $7.38 \pm 1.16 \mathrm{~Hz}$ and fractional $\mathrm{rms} 3 \%$ and $4.2 \%$ respectively. The low-frequency component is clearly the one responsible for the noise visible in Fig. A.3. The average power spectrum outside the QPO interval is quite different. It can be fitted with two zero-centered Lorentzians plus a broad QPO peak (see also Nespoli et al. 2003). The QPO has a centroid frequency of $v_{q}=6.71 \pm 0.14 \mathrm{~Hz}$ and a $\Delta=3.27 \pm 0.46$, for an integrated fractional rms of $2.2 \%$. The two noise components have characteristic frequencies $0.05 \pm 0.01 \mathrm{~Hz}$ and $0.94 \pm 0.19 \mathrm{~Hz}$ and fractional rms $1.6 \%$ and $1 \%$ respectively. Interestingly, there is a very narrow excess over the best-fit model at $6.6 \mathrm{~Hz}$, but an additional component is not required by the fits and nothing obvious is visible in the spectrogram. Here the type-B QPO power spectrum has a $0.1-64 \mathrm{~Hz}$ integrated fractional rms of $9.3 \%$, while for the broad QPO power spectrum it is $3.2 \%$. Since the hardness increases only slightly between the two intervals, notice that the $3.2 \%$ value occupies the position of the "type A" group, suggesting that the broad QPO is of type A (see below).

- Observation \#97: much later in the outburst, the sharp type-B QPO appears again in this observation. The first part of the observation $\sim 850 \mathrm{~s}$ shows no evidence of the QPO, which appears suddenly together with additional low-frequency noise (see Fig. A.4), with a variable centroid frequency once again correlated with the count rate. All these properties are consistent with those observed in previous observations, despite the fact that this observation takes place almost four months after Obs. \#43 (see Table 1). We produced a spectrogram with time resolution $16 \mathrm{~s}$ and averaged the power spectra corresponding to the presence and absence of QPO. The QPO is fitted with a Lorentzian with $v_{q}=5.71 \pm 0.02 \mathrm{~Hz}$ and a $\Delta=0.44 \pm 0.03$, for an integrated fractional $\mathrm{rms}$ of $6.5 \%$. It is accompanied by a clear second harmonic, and by a band-limited noise component with characteristic frequency $0.07 \pm 0.02 \mathrm{~Hz}$ and integrated fractional $\mathrm{rms} \sim 6 \%$. In the first part of the observation, when the QPO is not present, the average power spectrum is rather noisy: it can be fitted with a broad $(\Delta=4.05 \pm 1.15 \mathrm{~Hz})$ Lorentzian peak with a characteristic frequency of $7.41 \pm 0.43 \mathrm{~Hz}$ and an integrated fractional rms of $2.1 \%$, plus a band-limited noise with characteristic frequency $0.10 \pm 0.04 \mathrm{~Hz}$ and fractional $\mathrm{rms} 1.4 \%$. As for Obs. \#43, the integrated $0.1-64 \mathrm{~Hz}$ fractional rms is much lower when the type-B QPO is not present: with a value of $3.0 \%$, it brings the corresponding point in the "type A" group, although no significant QPO is present. 